\title{
Chiral Bifunctional Thiosquaramides as Organocatalysts in the Synthesis of Enantioenriched 3,3-Disubstituted Oxindoles
}

\author{
Patricia Rodríguez-Ferrer, ${ }^{[a]}$ Daniel Naharro, ${ }^{[a]}$ Alicia Maestro, ${ }^{[a]}$ José M. Andrés, ${ }^{*[a]}$ and Rafael \\ Pedrosa. ${ }^{*}[a]$
}

\begin{abstract}
Four novel chiral bifunctional thiosquaramides have been prepared from cyclopentyl dithiosquarates and diamines derived from natural L-Valine and L-tert-Leucine. The novel thiosquaramides have been tested as organocatalyst in the nitro-Michael addition of 3 -substituted oxindoles to different $\beta$-aryl substituted nitroalkenes. The reaction occurred easily in high yields and excellent stereoselectivities, showing that the novel organocatalysts are much more effective that their thioureas and squaramides homologs.
\end{abstract}

\section{Introduction}

Since the explosion of organocatalysis twenty years ago, a lot of small metal-free molecules able to promote stereoselective transformations have been developed. Among them, bifunctional organocatalysts that activate both the nucleophile by a basic appendage, and the electrophile by $\mathrm{H}$-bond formation behave as very efficient catalysts. The catalytic activity of these structures is dependent on the strength of the formed hydrogen bond, and the stereoselectivity on the nature of the chiral appendage. Searching for the modification of the acidity of the catalysts, chiral ureas, ${ }^{[1]}$ thioureas ${ }^{[2]}$ and squaramides ${ }^{[3]}$ have been the most common organocatalysts used in different stereoselective transformations.

Thiosquaramides are known more than fifty years ago, ${ }^{[4]}$ and they have more acidic character than squaramides. They are more soluble than squaramides in non-polar solvents as a consequence of less self-aggregation, and they have been calculated to be better organocatalysts than thioureas or squaramides. ${ }^{[5]}$ They have been used as anion transporters, ${ }^{[6]}$ or in the formation of complex of transition metal derivatives, ${ }^{[7]}$ but only very recently it has described a general synthesis of these substrates and used as organocatalysts. ${ }^{[8]}$ Our interest in developing bifunctional organocatalysts ${ }^{[9]}$ lead us to consider the preparation of novel bifunctional thiosquaramides and study if they were able to promote enantioselective additions of 3substituted oxindoles to nitrostyrenes.

The selection of oxindole derivatives as nucleophiles was motivated by the interest of the addition products as biological active substrates, ${ }^{[10]}$ and nitrostyrene because it is the most employed electrophile used in organocatalyzed conjugate additions, and the possibilities of transformations of the nitro functionality in the final products. The synthesis of enantioenriched 3,3-disubstituted oxindoles has received a lot of attention, ${ }^{[11]}$ but the most studied organocatalyzed transformation refers to the Michael addition of 3-substituted oxindoles to nitroolefins catalyzed by bifunctional thioureas. In that way, bifunctional thioureas bearing as chiral appendage cinchona alkaloids, ${ }^{[12]}$ 1,2-cyclohexanediamine, ${ }^{[13]}$ and 1,2diphenyl ethylenediamine ${ }^{[14]}$ have been used with high effectiveness.

Organocatalyst such as guanidines, ${ }^{[15]}$ phosphoramides, ${ }^{[16]}$ aminoindanol, ${ }^{[17]}$ phase transfer catalysts, ${ }^{[18]}$ chiral secondary amines, ${ }^{[19]}$ or bifunctional squaramides derived from 1,2cyclohexane diamine ${ }^{[20]}$ and quinine ${ }^{[21]}$ have been also used to promote the same stereoselective addition. Chiral bifunctional thioureas have been also employed in enantioselective additions of 3 -substituted oxindoles to maleimides, ${ }^{[22]}$ and unsaturated ketones or sulfones. ${ }^{[23]}$

Now we report on the synthesis of novel bifunctional chiral thiosquaramides with diamines derived from (L)-valine and (L)tert-leucine (Th-sq-la,b and Th-sq-Ila,b, Figure 1), and their unprecedented application as organocatalysts in the stereoselective synthesis of 3,3-disubstituted oxindoles with two quaternary-tertiary stereocenters. Known thioureas $(\mathbf{T h}-\mathbf{l a}, \mathbf{b})^{[24]}$ and squaramides $(\mathbf{s q}-\mathbf{l a}, \mathbf{b})^{[25]}$ have also tested in the same reaction for comparative purposes.

[a] Ms P. Rodríguez-Ferrer, Mr D. Naharro, Dr. A. Maestro, Prof. Dr. J.

M. Andrés, Prof. Dr. R. Pedrosa.

Instituto CINQUIMA and Departamento de Química Orgánica,

Facultad de Ciencias, Universidad de Valladolid, Paseo de Belén 7 .

47011-Valladolid. Spain.

E-mail: imandres@qo.uva.es; pedrosa@qo.uva.es

Homepage: http://sintesisasimetrica.blogs.uva.es/

Supporting information for this article is given via a link at the end of the document. 
<smiles>CC(C)C(CN(C)C)NC(=S)NCCc1ccccc1</smiles><smiles>CN(C)C[C@H](NC(=S)NCCc1ccccc1)C(C)(C)C</smiles>
Th-lb

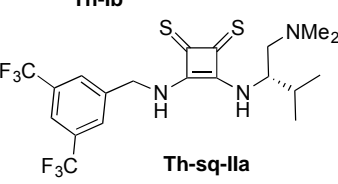
q-lb<smiles>Cc1cc(C(F)(F)F)cc(C(F)(F)F)c1</smiles>

Figure 1. Organocatalysts used in this work.

\section{Results and Discussion}

The synthesis of thiosquaramides was carried out in two steps from cyclopentyl dithiosquarate $\mathbf{1}^{[8 \mathrm{a}]}$ as summarized in Scheme 1. The reaction of dithiosquarate with 0.9 equivalents of benzylamine or 3,5-bis(trifluoromethyl)benzylamine, in dichloromethane (DCM) at room temperature, lead to the monodisplacement derivatives $2^{[8 a]}$ and 3 , respectively, in good yields. These hemidithiosquarates were transformed into the final chiral bifunctional thiosquaramides (Th-sq-la,b, and Th-sqIla,b) in moderate to good yields by reaction with 1.1 equivalents of diamines ${ }^{[26]}$ derived from natural L-Valine and L-tert-Leucine in $\mathrm{DCM}$ at room temperature. It is interesting to note that, contrary to the very well-known synthesis of unsymmetrically substituted squaramides, in our case it was not possible to alter the order of incorporation of two different amines to the thiosquaramides nucleus because the reaction of dithiosquarate 1 with the diamine derived from L-Valine leading to the monosubstitution compound $\mathbf{4}$ did not work (Scheme 1).

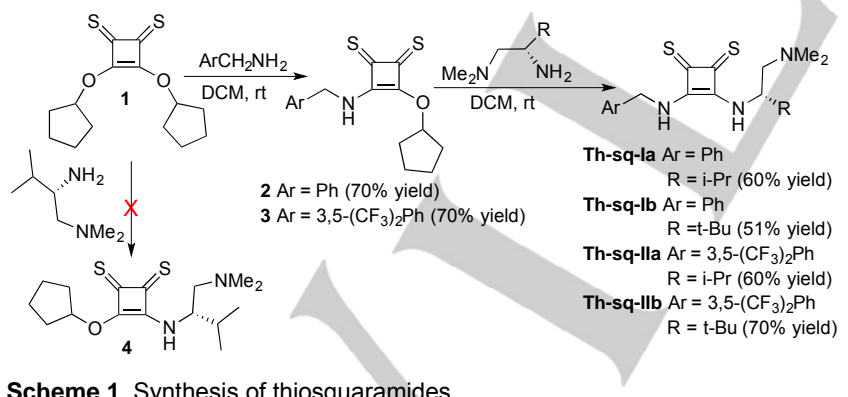

Scheme 1. Synthesis of thiosquaramides.

We initiated a study to calibrate the activity of the novel thiosquaramides against their thioureas (Th-la,b) and squaramides $(\mathbf{s q - l a , b )}$ homologs taken as reaction model the conjugate addition of 3-phenyl-susbtituted oxindoles (5a-d) to trans- -nitrostyrene (6a) (Table 1). First we focused our attention on the identification of the best reaction conditions by using 3-phenyl-1-methyl-substituted oxindole $\mathbf{5 a}$ as nucleophile, and $5 \mathrm{~mol} \%$ of thiosquaramide Th-sq-la as catalysts. The reaction occurred easily at room temperature in THF yielding the addition product 7 aa in excellent yield and good stereoselection (entry 1 in Table 1), and similar results were obtained when the reaction was carried out in toluene, although at expenses of increase the reaction time (entry 2). The same reaction in chloroform gave the addition product maintaining the stereoselectivity in much lower yield (entry 3 ), but the best yield, diastereo- and enantioselection were obtained in DCM (entry 4). Only a slight increase in the enantioselectivity was observed when the temperature was lowered to $-20^{\circ} \mathrm{C}$ (entry 5 ) or $-78^{\circ} \mathrm{C}$ (entry 6).

Table 1. Screening of the catalysts and optimization of the reaction conditions.<smiles>O=C1Nc2ccccc2C1c1ccccc1</smiles>

$5 a R^{1}=M e$

$5 b R^{1}=B n$

$5 c R^{1}=$ Boc

$5 d R^{1}=H$<smiles>[R]N1C(=O)N(C[N+](=O)[O-])c2ccccc2[C@H]1c1ccccc1</smiles>

7 aa $\mathrm{R}^{1}=\mathrm{Me}$

7 ba $\mathrm{R}^{1}=\mathrm{Bn}$

$7 \mathrm{ca} \mathrm{R}^{1}=\mathrm{Boc}$

$7 \mathrm{da} \mathrm{R}^{1}=\mathrm{H}$

\begin{tabular}{|c|c|c|c|c|c|c|c|}
\hline Entry & $\mathrm{R}^{1}$ & Catalyst & Solvent & $\begin{array}{l}\text { Time } \\
(\mathrm{h})\end{array}$ & $\begin{array}{c}\text { Product } \\
(\%)^{[a]}\end{array}$ & $d r^{[\mathrm{b}]}$ & $e r^{[c]}$ \\
\hline 1 & $\mathrm{Me}$ & Th-sq-la & THF & 2 & 7aa (92) & $89: 11$ & 93:7 \\
\hline 2 & $\mathrm{Me}$ & Th-sq-la & $\mathrm{PhMe}$ & 4 & $7 a a(84)$ & $87: 13$ & $93: 7$ \\
\hline 3 & $\mathrm{Me}$ & Th-sq-la & $\mathrm{CHCl}_{3}$ & 4 & $7 a a(58)$ & $85: 15$ & $92: 8$ \\
\hline 4 & $\mathrm{Me}$ & Th-sq-la & DCM & 2 & 7aa (97) & $91: 9$ & $96: 4$ \\
\hline $5^{[\mathrm{d}]}$ & $\mathrm{Me}$ & Th-sq-la & $\mathrm{DCM}$ & 2 & 7aa (99) & $90: 10$ & $97: 3$ \\
\hline $6^{[\mathrm{e}]}$ & $\mathrm{Me}$ & Th-sq-la & DCM & 5 & 7aa (98) & $93: 7$ & $98: 2$ \\
\hline 7 & $\mathrm{Me}$ & Th-la & DCM & 2 & 7aa (99) & $84: 16$ & $87: 11$ \\
\hline 8 & $\mathrm{Me}$ & Th-Ib & DCM & 1 & 7aa (67) & $78: 22$ & $88: 12$ \\
\hline 9 & $\mathrm{Me}$ & sq-la & DCM & 1 & 7aa (99) & $85: 15$ & $85: 15$ \\
\hline 10 & $\mathrm{Me}$ & & $\mathrm{DCM}$ & 3 & $7 a a(62)$ & $82: 18$ & $92: 8$ \\
\hline 11 & $\mathrm{Me}$ & Th- & $\mathrm{DCM}$ & 1.5 & $7 a a(81)$ & $87: 13$ & $96: 4$ \\
\hline 12 & $\mathrm{Me}$ & y-IIa & DCM & 0.5 & $7 a a(82)$ & $93: 7$ & $95: 5$ \\
\hline 13 & $\mathrm{Me}$ & Th-sq-Ilb & DCM & 1 & 7aa (76) & $86: 14$ & $97: 3$ \\
\hline 14 & $\mathrm{Bn}$ & Th-sq-la & DCM & 1 & $7 \mathrm{ba} \mathrm{(80)}$ & $95: 5$ & $97: 3$ \\
\hline 15 & $\mathrm{Bn}$ & Th-sq-Ib & $\overline{\mathrm{DCM}}$ & 1 & 7ba (98) & $93: 7$ & 99:1 \\
\hline 16 & $\mathrm{Bn}$ & Th-sq-IIb & DCM & 0.5 & $7 \mathbf{b a ~ ( 9 5 )}$ & $94: 6$ & $99: 1$ \\
\hline $17^{[f]}$ & $\mathrm{Bn}$ & Th-sq-Ilb & DCM & 2.5 & $7 \mathrm{ba} \mathrm{(87)}$ & $92: 8$ & $97: 3$ \\
\hline 18 & Boc & & $\mathrm{DCM}$ & 2 & 7ca (67) & $71: 29$ & $68: 32$ \\
\hline 19 & $\mathrm{H}$ & Th-sq-la & $\mathrm{DCM}$ & 1 & 7da (96) & $81: 19$ & $89: 11$ \\
\hline
\end{tabular}

[a] Values refer to pure isolated compound. [b] Measured by HPLC. [c

Determined by HPLC on a chiral column, and correspond to the major diastereoisomer. ${ }^{[\mathrm{dd}]}$ Reaction at $-20^{\circ} \mathrm{C}$. ${ }^{[e]}$ Reaction at $-78^{\circ} \mathrm{C}$. ${ }^{[\mathrm{fl}}$ Reaction performed with $1 \mathrm{~mol} \%$ of catalyst Th-sq-Ilb.

The effectiveness of all the catalysts was then tested under the best experimental conditions (rt, $5 \mathrm{~mol} \%$ of catalyst, DCM) (entries 7-13 in Table 1). From these data it is possible to conclude that catalysts derived from L-Valine (Th-la, sq-la, and Th-sq-la) are more diastereoselective, but less enantioselective than those derived from L-tert-Leucine (Th-lb, sq-lb, and Th-sqlb) (compare entries 7, 9, 11, and 12 versus $8,10,4$ and 13, respectively), and that thiosquaramides are, in general more effective catalysts than thioureas or squaramides in this reaction. To study the influence of the substituent on the nitrogen atom we reacted different substituted oxindoles (5b-d) with trans- $\beta$ nitrostyrene in the presence of thiosquaramides as the best catalysts (entries 14-19). Interestingly, unsubstituted oxindole 5d 
WILEY-VCH

easily reacted leading to $\mathbf{7 d a}$, but with lower stereocontrol than the methyl-substituted substrate $\mathbf{5 a}$ (compare entries 19 and 4). The same fact was observed for the reaction of N-Bocsubstituted derivative $\mathbf{5 c}$, in that case the addition product $7 \mathrm{ca}$ was obtained in moderate yield and very poor stereocontrol (entry 18). On the contrary, the reaction of $N$-benzyl-substituted oxindole $5 \mathbf{b}$ was the most stereoselective reaction independently of the thiosquaramide used as catalyst (entries 14-16). Interestingly, only a slight increase in the reaction time, and lowering diastereoselectivity was observed when the ratio of catalyst Th-sq-Ilb can be decreased to $1 \mathrm{~mol} \%$ (entry 17 ).

The influence in the reaction of the electronic nature of the substituent at the oxindole and nitroolefin aromatic rings was studied by using different 3-aryl-1-benzyl-5-substituted oxindoles $\mathbf{5 b}-\mathbf{I}$ as nucleophiles, $\beta$-aryl nitroolefins $\mathbf{6 a - f}$ as electrophiles and Th-sq-Ilb as the best catalyst at room temperature (Table 2). The electronic character of the nitroolefin has little or no influence on the addition process. Both nitrostyrenes bearing electron withdrawing- $(\mathbf{6 b}, \mathbf{c})$ or donating groups $(\mathbf{6 d})$ behave in a similar way that the parent nitrostyrene $(\mathbf{6 a})$, yielding the addition products in excellent yield (81-97\%) and stereoselection (dr 92:8-94:6, er 97:3-99:1) (compare entries 1-3 in Table 2 versus entry 16 in Table 1). The reaction of the naphthyl derivative $6 \mathrm{e}$ was slower, although maintaining the excellent yield and stereoselectivity (entry 4 in Table 2), but the furyl nitroolefin $6 \mathrm{f}$ leaded to the addition product $\mathbf{7 b f}$ in good enantioselection and moderate yield and diastereoselectivity (entry 5)

Table 2. Scope of the reaction for different substituted $\mathrm{N}$-benzyloxindoles with nitroolefins.<smiles>[R]c1cc([R])c2c(c1)C([R])C(=O)N2Cc1ccccc1</smiles>

$5 \mathbf{b} R^{2}=R^{3}=H, R=P h$ 5e $R^{2}=M e, R^{3}=H, R=P h$ $5 f R^{2}=F, R^{3}=H, R=P h$ $\mathbf{5 g} \mathrm{R}^{2}=\mathrm{Cl}, \mathrm{R}^{3}=\mathrm{H}, \mathrm{R}=\mathrm{Ph}$ $5 h R^{2}=B r, R^{3}=H, R=P h$ 5i $\mathrm{R}^{2}=\mathrm{MeO}, \mathrm{R}^{3}=\mathrm{H}, \mathrm{R}=\mathrm{Ph}$ $5 j \mathrm{R}^{2}=\mathrm{H}, \mathrm{R}^{3}=\mathrm{CF}_{3}, \mathrm{R}=\mathrm{Ph}$ $5 \mathbf{R}^{2}=\mathrm{R}^{3}=\mathrm{H}, \mathrm{R}=4-\mathrm{CF}_{3} \mathrm{C}_{6} \mathrm{H}_{4}$ $5 \mathrm{R}^{2}=\mathrm{R}^{3}=\mathrm{H}, \mathrm{R}=4-\mathrm{MeOC}_{6} \mathrm{H}_{4}$ $5 m R^{2}=R^{3}=H, R=M e$

\begin{tabular}{|c|c|c|c|c|c|}
\hline Entry $^{[\mathrm{a}]}$ & Reagents & Time $(\mathrm{h})$ & $\begin{array}{c}\text { Product } \\
(\%)^{[\mathrm{b}]}\end{array}$ & $d r^{[\mathrm{c}]}$ & $\mathrm{er}^{[\mathrm{c}]}$ \\
\hline 1 & $\mathbf{5 b / 6 b}$ & 0.5 & $\mathbf{7 b b}(81)$ & $94: 6$ & $99: 1$ \\
\hline 2 & $\mathbf{5 b / 6 c}$ & 2 & $\mathbf{7 b c}(97)$ & $93: 7$ & $99: 1$ \\
\hline 3 & $\mathbf{5 b / 6 d}$ & 0.5 & $\mathbf{7 b d}(81)$ & $92: 8$ & $97: 3$ \\
\hline 4 & $\mathbf{5 b / 6 e}$ & 5 & $\mathbf{7 b e}(98)$ & $93: 7$ & $98: 2$ \\
\hline 5 & $\mathbf{5 b / 6 f}$ & 1 & $\mathbf{7 b f}(73)$ & $84: 16$ & $97: 3$ \\
\hline 6 & $\mathbf{5 e / 6 a}$ & 0.5 & $\mathbf{7 e a}(84)$ & $89: 11$ & $98: 2$ \\
\hline 7 & $\mathbf{5 f / 6 a}$ & 0.5 & $\mathbf{7 f a}(95)$ & $88: 12$ & $97: 3$ \\
\hline $8^{[\mathrm{d}]}$ & $\mathbf{5 f / 6 a}$ & 3 & $\mathbf{7 f a}(95)$ & $92: 8$ & $97: 3$ \\
\hline 9 & $\mathbf{5 g / 6 a}$ & 0.5 & $\mathbf{7 g a}(92)$ & $86: 14$ & $95: 5$ \\
\hline
\end{tabular}

\begin{tabular}{|c|c|c|c|c|c|}
\hline $10^{[\mathrm{d}]}$ & $\mathbf{5 g} / \mathbf{6 a}$ & 3 & $\mathbf{7 g a}(82)$ & $90: 10$ & $97: 3$ \\
\hline 11 & $\mathbf{5 h / 6 a}$ & 0.5 & $\mathbf{7 h a}(91)$ & $82: 18$ & $93: 7$ \\
\hline $12^{[\mathrm{e}]}$ & $\mathbf{5 h / 6 a}$ & 0.5 & $\mathbf{7 h a}(\mathbf{7 2})^{[\mathrm{e}]}$ & $97: 3^{[\mathrm{e}]}$ & $97: 3^{[\mathrm{e}]}$ \\
\hline 13 & $\mathbf{5 i} / \mathbf{6 a}$ & 0.5 & $\mathbf{7 i a}(71)$ & $97: 3$ & $99: 1$ \\
\hline 14 & $\mathbf{5 j} / \mathbf{6 a}$ & 0.5 & $\mathbf{7 j a}(96)$ & $60: 40$ & $91: 9$ \\
\hline $15^{[\mathrm{d}]}$ & $\mathbf{5 j} / \mathbf{6 a}$ & 3 & $\mathbf{7 j a}(98)$ & $81: 19$ & $97: 3$ \\
\hline 16 & $\mathbf{5 k} / \mathbf{6 a}$ & 0.5 & $\mathbf{7 k a}(98)$ & $90: 10$ & $98: 2$ \\
\hline 17 & $\mathbf{5 l} \mathbf{6 a}$ & 0.5 & $\mathbf{7 l a}(63)$ & $99: 1$ & $>99:<1$ \\
\hline $18^{[\mathrm{f}]}$ & $\mathbf{5 m} / \mathbf{6 a}$ & $\mathbf{2 7}$ & $\mathbf{7 m a}(83)$ & $\mathbf{7 9 : 2 1}$ & $95: 5$ \\
\hline
\end{tabular}

[a] The reactions were carried out with $0.15 \mathrm{mmol}$ of oxindole, $0.30 \mathrm{mmol}$ of nitroalkene, and $5 \mathrm{~mol} \%$ of catalysts in $0.2 \mathrm{~mL}$ of DCM. ${ }^{[b]}$ Values refer to pure compounds after column chromatography. ${ }^{[c]}$ Measured by HPLC on a chiral column. [d] The reaction was carried out at $-40^{\circ} \mathrm{C}$. ${ }^{[e]}$ The reaction was scaled up to $1.5 \mathrm{mmol}$ of nucleophile, and yield, $d r$, and er was determined after recrystallization. ${ }^{[f]}$ The reaction was carried out with $20 \mathrm{~mol} \%$ of catalyst.

The effect of a substituent at the aromatic ring in the oxindole was studied by reacting compounds $5 \mathrm{e}-\mathrm{i}$, with a substituent at $\mathrm{C}$ 5 , and the C-7 substituted oxindole $5 \mathbf{j}$, with nitrostyrene $\mathbf{6 a}$ (entries 6-15 in Table 2). Interestingly, the presence of a methyl group (5e) or a halogen (5f-h) at C-5 in the oxindole nucleus does not modify the reactivity of the nucleophile, although decreased the diastereoselection maintaining the excellent enantioselectivity (entries 6, 7, 9, and 11 in Table 2). To demonstrate the interest of the process, the reaction of $5 \mathrm{~h}$ with $6 a$ was tenfold scaled up with similar results, but increasing the stereoselection to $97: 3 d r$ and 97:3 er, after recrystallization of 7 ha (entry 12 in Table 2). On the other hand, the stereoselection was recovered to excellent levels when the reaction was carried out at low temperature $\left(-40^{\circ} \mathrm{C}\right)$ for longer reaction times (entries $8,10)$. The only exception to that general behavior corresponds to 5 -methoxy oxindole $\mathbf{5 i}$, which gave the addition product $7 \mathbf{i a}$ in lower yield although very good diastereo- and enantioselection (entry 13).

Oxindole $5 \mathrm{j}$, with an electron withdrawing substituent at C-7 also reacted easily with nitrostyrene, but yielding $7 \mathbf{j a}$ in very poor diastereoselection. Fortunately, both the diastereo- and enantioselectivity were improved at low temperature (compare entries 14 and 15 in Table 2). On the contrary, the electronic nature of a substituent at C-4 in the aryl group attached to C-3 in the oxindole plays an important role in the reaction. Thus, compound $\mathbf{5 k}$ with a $4^{\prime}$-trifluromethyl phenyl substituent at C-3 easily reacted with nitrostyrene yielding $7 \mathbf{k a}$ in good diastereoselectivity and excellent yield and enantioselection but 5I, with an electron-donating $(\mathrm{MeO})$ group at the same position gave the addition product 7la with total stereoselectivity in moderate yield (entries 16, 17 in Table 2).

The absolute stereochemistry of the major enantiomer was determined as $(R, R)$ by XR-diffraction analysis for $\mathbf{7 h a} \mathbf{h}^{[27]}$ (Figure 2 ), and extended for all the addition products by analogy. 


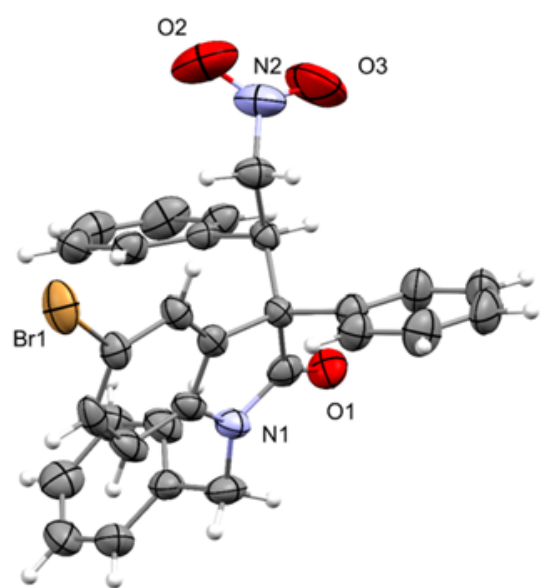

Figure 2. ORTEP representation of the structure of 7 ha determined by X-ray diffraction analysis. The thermal ellipsoids are shown at $50 \%$ probability.

Furthermore, in an attempt to increase the scope of the process we study the reaction of the less acidic 3-methyl substituted oxindole $5 \mathrm{~m}$ with nitrostyrene. As expected, the reaction was really slow $(120 \mathrm{~h})$ when $5 \mathrm{~mol} \%$ of catalyst was used, but it was completed in $24 \mathrm{~h}$ with good yield, moderate diastereoselection (dr 79:21), and excellent enantioselection (er 95:5) by increasing the ratio of catalyst Th-sq-Ilb to $20 \mathrm{~mol} \%$ (entry 18 in Table 2). As an example of the synthetic utility of the reaction we have prepared, in two steps, the enantioenriched spirooxindolyl lactam 8, with two contiguous quaternary-tertiary stereocenters as summarized in Scheme 2. These class of compounds have recently received a great synthetic interest ${ }^{[28]}$ because their important biological properties. ${ }^{[29]}$<smiles>CCOC(=O)CC1C(=O)Nc2ccccc21</smiles>

$5 n$

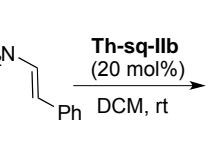

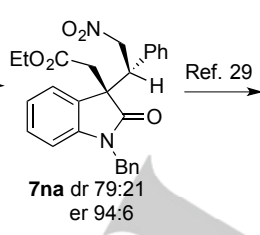

er $94: 6$
$\mathrm{O} \underset{\mathrm{N}}{\mathrm{H}}$<smiles>CCC1(CC)C(=O)Nc2ccccc21</smiles>
$\mathrm{Bn}$ $883 \%$, er $94: 6$
Scheme 2. Two steps synthesis of spirooxindolyl lactam $\mathbf{8}$.

A solution of $5 \mathbf{n}$ and trans- $\beta$-nitrostyrene (6a) in DCM was stirred at r.t. for $24 \mathrm{~h}$ in the presence of $20 \mathrm{~mol} \%$ of Th-sq-Ilb leading to the addition product $\mathbf{7 n a}$ in good yield $(82 \%)$, moderate diastereoselectivity ( $d r$ 79:21), and excellent enantioselection (ee 94:6). The major diastereoisomer was isolated by flash chromatography, and transformed into spirooxindolyl lactam 8 $(83 \%)$ by reduction of the nitro group with $\mathrm{Zn} / \mathrm{HOAc},{ }^{[30]}$ and in situ lactamization. HPLC on a chiral phase shown that compound $\mathbf{8}$ was obtained with the same enantiomeric ratio than 7na, showing that no epimerization occurred during the reduction-lactamization process.

\section{Conclusions}

We have prepared four novel thiosquaramides in two steps by sequential transamination of cyclopentyl dithiosquarate with benzylamine or 3,5-bis(trifluoromethyl)benzylamine, and chiral diamines derived from L-Valine or L-tert-Leucine. These thiosquaramides have been tested as organocatalysts in the stereoselective addition of 3-substituted oxindoles to different trans- $\beta$-aryl nitroolefins. The reported data show that the nitroMichael addition occurred in high yield and diastereoselectivity, and excellent enantioselection. The novel organocatalysts provide much better results than their thiourea or squaramide counterparts. The described strategy has been applied as a key step in the synthesis of an example of enantioenriched biologically active spirooxindolyl lactam.

\section{Experimental Section}

General

New compounds were characterized by ${ }^{1} \mathrm{H}$ NMR $(500 \mathrm{MHz}),{ }^{13} \mathrm{C}$ NMR (126 MHz), high-resolution mass spectrometry (Agilent 5973, ESI-QTOF), IR spectroscopy (Perkin-Elmer Spectrum One FT-IR spectrometer), and elemental analysis (Elemental Analysis Center of the Complutense University of Madrid, using a Perkin Elmer $2400 \mathrm{CHN}$ ). Chemical shifts for protons and carbons are reported in ppm from TMS with the residual $\mathrm{CHCl}_{3}$ resonance for protons, and carbon resonance of the solvent for carbons as internal references. Data are reported as follows: chemical shift, multiplicity $(\mathrm{s}=$ singlet, $\mathrm{d}=$ doublet, $\mathrm{t}=$ triplet, $\mathrm{q}=$ quartet, $\mathrm{m}=$ multiplet, $b r=$ broad), coupling constants in Hertz, and integration.

Specific rotations (concentration given in g per $100 \mathrm{~mL}$ ) were measured on a Perkin-Elmer 341 digital polarimeter using a $5-\mathrm{mL}$ cell with a $1-\mathrm{dm}$ path length, and sodium lamp. Melting points were obtained with open capillary tubes and are uncorrected. TLC analysis was performed on glass-backed plates coated with silica gel 60 and an $F_{254}$ indicator, and visualized by either UV irradiation or by staining with phosphomolybdic acid solution, and Column chromatography was carried out using silica gel (230-240 mesh).

Chiral HPLC analysis was performed on a JASCO HPLC system (JASCO PU-2089 pump and UV-2075 UV/Vis detector) and on HewlettPackard 1090 Series II instrument equipped with a quaternary pump, using a Chiralpak IA, Lux-amylose-1 and Lux-i-Amylose-1 analytical columns $(250 \times 4.6 \mathrm{~mm})$. UV detection was monitored at 220 or at 254 $\mathrm{nm}$

Commercially available organic and inorganic compounds were used without further purification. Solvents were dried and stored over microwave-activated $4 \AA$ molecular sieves. Known compounds $\mathbf{1}^{[8 \mathrm{a}]}, \mathbf{2},{ }^{\text {[8a] }}$ $\mathbf{5 a - b}, \mathbf{d}, \mathbf{I},{ }^{[31]} \mathbf{5 c},{ }^{[32]} \mathbf{5 e ,},{ }^{[33]} \mathbf{5 m},{ }^{[34]}$ and $\mathbf{5 n}^{[35]}$ have been prepared as described in the literature.

3-((3,5-Bis(trifluoromethyl)benzyl)amino)-4-(cyclopentoxy)cyclobut3-ene-1,2-dithione (3). To a solution of 1 (1.20 g, $4.25 \mathrm{mmol}, 1.0$ equiv) in dry $\mathrm{CH}_{2} \mathrm{Cl}_{2}(20 \mathrm{~mL})$ was added 3,5-bis(trifluoromethyl)benzylamine ( $0.42 \mathrm{~mL}, 3.83 \mathrm{mmol}, 0.90$ equiv) at $0{ }^{\circ} \mathrm{C}$ and the resulting solution was stirred for $15 \mathrm{~min}$ at that temperature, and then $15 \mathrm{~min}$ at room temperature. The solution was transferred to a silica gel column and quickly eluted with $\mathrm{CH}_{2} \mathrm{Cl}_{2}$ to afford $3(1.18 \mathrm{~g}, 2.68 \mathrm{mmol}, 70 \%)$ as an 
amorphous yellow solid; \%), m.p. $84-85^{\circ} \mathrm{C}$. The compound exists as a mixture of two rotamers in DMSO at room temperature in a ratio 0.61:0.37. Major rotamer: ${ }^{1} \mathrm{H}$ NMR $\left(500 \mathrm{MHz}\right.$, DMSO-d $\left.\mathrm{d}_{6}\right) \delta=10.14(\mathrm{t}, J=$ $6.0 \mathrm{~Hz}, 1 \mathrm{H}), 8.10(\mathrm{~s}, 1 \mathrm{H}), 8.04(\mathrm{~s}, 2 \mathrm{H}), 6.34(\mathrm{~m}, 1 \mathrm{H}), 4.76(\mathrm{~d}, J=6.0 \mathrm{~Hz}$, $2 \mathrm{H}), 2.00-1.84(\mathrm{~m}, 4 \mathrm{H}), 1.77-1.57(\mathrm{~m}, 4 \mathrm{H}) \mathrm{ppm}$. Minor rotamer: ${ }^{1} \mathrm{H}$ NMR $\left(500 \mathrm{MHz}, \mathrm{DMSO}-\mathrm{d}_{6}\right) \delta 10.07$ (t, $J=6.0 \mathrm{~Hz}, 1 \mathrm{H}$ ), $8.10(\mathrm{~s}, 1 \mathrm{H}), 8.04(\mathrm{~s}$, 2H), $6.29(\mathrm{~m}, 1 \mathrm{H}), 5.37(\mathrm{~d}, J=6.0 \mathrm{~Hz}, 2 \mathrm{H}), 2.00-1.84(\mathrm{~m}, 4 \mathrm{H}), 1.77-1.57$ (m, 4H) ppm. ${ }^{13} \mathrm{C}$ NMR (126 MHz, DMSO-d 6 ) $\delta=217.9,217.7,206.8$, 206.8, 183.5, 182.8, 176.1, 173.8, 141.1, 141.0, 130.9 (q, ${ }^{2} \mathrm{JC}_{\mathrm{C}-\mathrm{F}}=32.9$ $\mathrm{Hz}), 130.9\left(\mathrm{q},{ }^{2} \mathrm{~J}_{\mathrm{C}-\mathrm{F}}=32.9 \mathrm{~Hz}\right), 129.5(\mathrm{~m}), 129.2(\mathrm{~m}), 123.7\left(\mathrm{q},{ }^{1} \mathrm{~J}_{\mathrm{C}-\mathrm{F}}=\right.$ $272.8 \mathrm{~Hz}), 122.0(\mathrm{~m}), 87.9,87.8,47.5,45.5,35.4,34.2,34.1$., 23.7, 23.5, $23.4 \mathrm{ppm}$. IR (ATR): $\tilde{v}=3242,2964,1693,1514,1415,681 \mathrm{~cm}^{-1}$. HRMS (ESI-QTOF) $\mathrm{m} / \mathrm{z}:[\mathrm{M}+\mathrm{H}]^{+}$Calcd. for $\mathrm{C}_{18} \mathrm{H}_{15} \mathrm{~F}_{6} \mathrm{NNaOS}_{2}$ 462.0391; Found 462.0395

(S)-3-(Benzylamino)-4-((1-(dimethylamino)-3-methylbutan-2-

yl)amino)cyclobut-3-ene-1,2-dithione (Th-sq-la). To a solution of 2 (300 mg, $0.99 \mathrm{mmol}$ ) in anhydrous $\mathrm{CH}_{2} \mathrm{Cl}_{2}(4 \mathrm{~mL})$ was added dropwise a solution of $(S)-N^{1}, N^{1}, 3$-trimethylbutane-1,2-diamine ${ }^{[26]}$ (142 mg, 1.09 mmol, 1.1 equiv) in anhydrous $\mathrm{CH}_{2} \mathrm{Cl}_{2}(0.5 \mathrm{~mL})$ at $0{ }^{\circ} \mathrm{C}$ and the resulting solution was stirred for $5 \mathrm{~min}$ at that temperature, and then $36 \mathrm{~h}$ at room temperature. The solvent was removed under vacuum and immediately purified by flash column chromatography $\left(\mathrm{CH}_{2} \mathrm{Cl}_{2} / \mathrm{MeOH}: 50: 1\right)$ to afford catalyst Th-sq-la as a brown solid (206 mg, $0.60 \mathrm{mmol}, 60 \%)$; m.p. 129$130{ }^{\circ} \mathrm{C} .[\mathrm{\alpha}]_{\mathrm{D}}{ }^{23}=-94.3\left(c=0.30, \mathrm{CHCl}_{3}\right) .{ }^{1} \mathrm{H}$ NMR $\left(500 \mathrm{MHz}, \mathrm{DMSO}-\mathrm{d}_{6}\right) \delta$ $=9.02(\mathrm{br}, 1 \mathrm{H}), 8.57(\mathrm{br}, 1 \mathrm{H}), 7.43-7.30(\mathrm{~m}, 5 \mathrm{H}), 5.36(\mathrm{~d}, J=13.2 \mathrm{~Hz}$, $1 \mathrm{H}), 5.26(\mathrm{~d}, J=13.2 \mathrm{~Hz}, 1 \mathrm{H}), 5.18(\mathrm{br}, 1 \mathrm{H}), 2.57(\mathrm{br}, 1 \mathrm{H}), 2.39(\mathrm{br}, 1 \mathrm{H})$, $2.25(\mathrm{br}, 6 \mathrm{H}), 1.85(\mathrm{br}, 1 \mathrm{H}), 0.90(\mathrm{~d}, J=6.8 \mathrm{~Hz}, 3 \mathrm{H}), 0.86(\mathrm{~d}, J=6.8 \mathrm{~Hz}$, $3 \mathrm{H}) \mathrm{ppm} .{ }^{13} \mathrm{C}$ NMR (126 MHz, DMSO-d 6 ) $\delta=204.4,204.0,171.3,170.5$, 137.9, 129.3, 128.6, 128.3, 61.1, 55.5, 46.5, 45.6, 31.2, 19.4, 17.1 ppm. IR (ATR): $\tilde{v}=3170,2958,1703,1569,1453,732,696 \mathrm{~cm}^{-1}$. HRMS (ESIQTOF) m/z: $[M+\mathrm{H}]^{+}$Calcd. for $\mathrm{C}_{28} \mathrm{H}_{26} \mathrm{~N}_{3} \mathrm{~S}_{2} 348.1559$; Found 348.1563 . (S)-3-(Benzylamino)-4-((1-(dimethylamino)-3,3-dimethylbutan-2yl)amino) cyclobut-3-ene-1,2-dithione (Th-sq-Ib). To a solution of 2 (300 mg, $0.99 \mathrm{mmol}, 1.0$ equiv) in anhydrous $\mathrm{CH}_{2} \mathrm{Cl}_{2}(4 \mathrm{~mL}$ ) was added dropwise a solution of (S)- $N^{1}, N^{1}, 3,3$-tetramethylbutane-1,2-diamine ${ }^{[26]}$ (157 mg, $1.09 \mathrm{mmol}, 1.1$ equiv) in dry $\mathrm{CH}_{2} \mathrm{Cl}_{2}(0.5 \mathrm{~mL})$ at $0{ }^{\circ} \mathrm{C}$ and the resulting solution was stirred for $5 \mathrm{~min}$ at that temperature, and then $36 \mathrm{~h}$ at room temperature. The solvent was removed under vacuum and immediately purified by flash column chromatography $\left(\mathrm{CH}_{2} \mathrm{Cl}_{2} / \mathrm{MeOH}\right.$ : 50:1) to afford catalyst Th-sq-lb as a brown solid (182 mg, $0.505 \mathrm{mmol}$, $51 \%)$; m.p. $138-139{ }^{\circ} \mathrm{C} .[\alpha]_{\mathrm{D}^{23}}=-77.3\left(c=0.30, \mathrm{CHCl}_{3}\right) .{ }^{1} \mathrm{H}$ NMR $(500$ $\left.\mathrm{MHz}, \mathrm{DMSO}-\mathrm{d}_{6}\right) \delta=8.85(\mathrm{br}, 1 \mathrm{H}), 8.51(\mathrm{br}, 1 \mathrm{H}), 7.43-7.32(\mathrm{~m}, 5 \mathrm{H}), 5.36$ $(\mathrm{d}, J=14.3 \mathrm{~Hz}, 1 \mathrm{H}), 5.28(\mathrm{~d}, J=14.2 \mathrm{~Hz}, 1 \mathrm{H}), 5.06(\mathrm{br}, 1 \mathrm{H}), 2.48(\mathrm{~m}, 2 \mathrm{H})$ 2.18 (br, 6H), 0.91 (s, 9H) ppm. ${ }^{13} \mathrm{C} \mathrm{NMR}\left(126 \mathrm{MHz}, \mathrm{DMSO}-\mathrm{d}_{6}\right) \delta=204.5$ 204.0, 171.4, 170.4, 137.9, 129.3, 128.7, 128.3, 59.3, 58.7, 46.5, 45.6, 34.4, 26.4 ppm. IR (ATR): $\tilde{v}=3172,2958,1704,1569,1477,727,696$ $\mathrm{cm}^{-1}$. HRMS (ESI-QTOF) m/z: $[M+\mathrm{H}]^{+}$Calcd. for $\mathrm{C}_{19} \mathrm{H}_{28} \mathrm{~N}_{3} \mathrm{~S}_{2} 362.1719$; Found 362.1721.

(S)-3-((3,5-Bis(trifluoromethyl)benzyl)amino)-4-((1-(dimethylamino)3-methylbutan-2-yl)amino)cyclobut-3-ene-1,2-dithione (Th-sq-lla). To a solution of 3 (439 mg, $1.0 \mathrm{mmol}$ ) in dry $\mathrm{CH}_{2} \mathrm{Cl}_{2}(4 \mathrm{~mL})$ was added dropwise a solution of $(S)-N^{1}, N^{1}, 3$-trimethylbutane-1,2-diamine ${ }^{[26]}$ (143 $\mathrm{mg}, 1.1 \mathrm{mmol}, 1.1$ equiv) in dry $\mathrm{CH}_{2} \mathrm{Cl}_{2}(0.5 \mathrm{~mL})$ at $0{ }^{\circ} \mathrm{C}$ and the resulting solution was stirred for $5 \mathrm{~min}$ at that temperature, and then $20 \mathrm{~h}$ at room temperature. The solvent was removed under vacuum and immediately purified by flash column chromatography $\left(\mathrm{CH}_{2} \mathrm{Cl}_{2} / \mathrm{MeOH}: 50: 1\right)$ to afford catalyst Th-sq-lla as a brown solid $(290 \mathrm{mg}, 0.6 \mathrm{mmol}, 60 \%)$, m.p. 123$124{ }^{\circ} \mathrm{C} .[\alpha]_{\mathrm{D}}{ }^{23}=-24.0\left(c=0.20, \mathrm{CHCl}_{3}\right) .{ }^{1} \mathrm{H}$ NMR $\left(500 \mathrm{MHz}, \mathrm{DMSO}-\mathrm{d}_{6}\right) \delta$ $=9.13(\mathrm{br}, 1 \mathrm{H}), 8.77(\mathrm{br}, 1 \mathrm{H}), 8.16(\mathrm{~s}, 2 \mathrm{H}), 8.05(\mathrm{~s}, 1 \mathrm{H}), 5.54(\mathrm{~d}, J=15.1$ $\mathrm{Hz}, 1 \mathrm{H}), 5.48(\mathrm{~d}, J=15.1 \mathrm{~Hz}, 1 \mathrm{H}), 5.19(\mathrm{br}, 1 \mathrm{H}), 2.64(\mathrm{br}, 1 \mathrm{H}), 2.50(\mathrm{br}$, $1 \mathrm{H}), 2.30(\mathrm{br}, 6 \mathrm{H}), 1.87-1.85(\mathrm{~m}, 1 \mathrm{H}), 0.90(\mathrm{~d}, J=6.8 \mathrm{~Hz}, 3 \mathrm{H}), 0.87(\mathrm{~d}, J$ $=6.8 \mathrm{~Hz}, 3 \mathrm{H}) \mathrm{ppm} .{ }^{13} \mathrm{C} \mathrm{NMR}\left(126 \mathrm{MHz}, \mathrm{DMSO}-\mathrm{d}_{6}\right) \delta=205.2,203.8$, 171.4, 171.1, 141.6, 131.0 (q, ${ }^{2} \mathrm{JC}_{\mathrm{C}-\mathrm{F}}=33.1 \mathrm{~Hz}$ ), $129.3(\mathrm{~m}), 123.7$ (q, ${ }^{1} \mathrm{JC}_{\mathrm{C}-\mathrm{F}}$ $=272.9 \mathrm{~Hz}), 121.9(\mathrm{~m}), 60.9,55.5,45.3,31.2,19.3,17.2 \mathrm{ppm}$. IR (ATR): $\tilde{v}=3237,3166,2964,1702,1575,1462,704,681 \mathrm{~cm}^{-1}$. HRMS (ESIQTOF) m/z: $[M+H]^{+}$Calcd. for $\mathrm{C}_{20} \mathrm{H}_{24} \mathrm{~F}_{6} \mathrm{~N}_{3} \mathrm{~S}_{2}$ 484.1310; Found 484.1328.

(S)-3-((3,5-Bis(trifluoromethyl)benzyl)amino)-4-((1-(dimethylamino)3,3-dimethylbutan-2-yl)amino)cyclobut-3-ene-1,2-dithione (Th-sq-llb). To a solution of $3(439 \mathrm{mg}, 1.0 \mathrm{mmol})$ in dry $\mathrm{CH}_{2} \mathrm{Cl}_{2}(4 \mathrm{~mL})$ was added dropwise a solution of $(S)-N^{1}, N^{1}, 3,3$-tetramethylbutane-1,2-diamine ${ }^{[26]}$ (159 mg, $1.1 \mathrm{mmol}, 1.1$ equiv) in dry $\mathrm{CH}_{2} \mathrm{Cl}_{2}(0.5 \mathrm{~mL})$ at $0{ }^{\circ} \mathrm{C}$ and the resulting solution was stirred for $5 \mathrm{~min}$ at that temperature, and then $3.5 \mathrm{~h}$ at room temperature. The solvent was removed under vacuum and immediately purified by flash column chromatography $\left(\mathrm{MeOH} / \mathrm{CH}_{2} \mathrm{Cl}_{2}\right.$ 1:50) to afford catalyst Th-sq-Ilb as a brown solid $(348 \mathrm{mg}, 0.70 \mathrm{mmol}$, $70 \%$; m.p. $164-165{ }^{\circ} \mathrm{C}[\alpha]_{D}{ }^{23}=-22.0\left(c=0.10, \mathrm{CHCl}_{3}\right) .{ }^{1} \mathrm{H}$ NMR $(500$ $\left.\mathrm{MHz}, \mathrm{DMSO}-\mathrm{d}_{6}\right) \delta=9.02(\mathrm{br}, 1 \mathrm{H}), 8.71(\mathrm{br}, 1 \mathrm{H}), 8.16(\mathrm{~s}, 2 \mathrm{H}), 8.08(\mathrm{~s}$, $1 \mathrm{H}), 5.55(\mathrm{~d}, J=15.0 \mathrm{~Hz}, 1 \mathrm{H}), 5.47(\mathrm{~d}, J=15.0 \mathrm{~Hz}, 1 \mathrm{H}), 5.06$ (br, $1 \mathrm{H})$, $2.48(\mathrm{~m}, 2 \mathrm{H}), 2.23(\mathrm{br}, 6 \mathrm{H}), 0.91(\mathrm{~s}, 9 \mathrm{H}) \mathrm{ppm} .{ }^{13} \mathrm{C} \mathrm{NMR}(126 \mathrm{MHz}$, DMSO-d 6 ) $\delta=205.5,205.5,171.4,171.0,141.7,131.0\left(\mathrm{q},{ }^{2} \mathrm{JC}_{\mathrm{C}-\mathrm{F}}=32.6\right.$ $\mathrm{Hz}), 129.5(\mathrm{~m}), 129.0(\mathrm{~m}), 123.7$ (q, $\left.{ }^{1} \mathrm{JC}_{\mathrm{C}-\mathrm{F}}=273.0 \mathrm{~Hz}\right) 122.1(\mathrm{~m}), 59.2$, $58.8,45.6,45.3,34.4,26.4 \mathrm{ppm}$. IR (ATR): $\tilde{v}=3242,3162,2964,1707$, 1575, 1476, 704, $681 \mathrm{~cm}^{-1}$. HRMS (ESI-QTOF) m/z: $[M+\mathrm{H}]^{+}$Calcd. for $\mathrm{C}_{21} \mathrm{H}_{26} \mathrm{~F}_{6} \mathrm{~N}_{3} \mathrm{~S}_{2}$ 498.1467; Found 498.1485.

General procedure for the synthesis of oxindoles $5 f-k$. The synthesis of these compounds was carried out in two steps as previously described. ${ }^{[31]}$ To a cooled solution $\left(0{ }^{\circ} \mathrm{C}\right)$ of the corresponding benzylisatin $(17.0 \mathrm{mmol})$ in THF $(55 \mathrm{~mL})$ was added a solution of the corresponding arylmagnesium bromide in $\mathrm{Et}_{2} \mathrm{O}$ ( $20.4 \mathrm{mmol}, 1.2$ equiv) under nitrogen atmosphere. The mixture was warmed to room temperature and stirred for $30 \mathrm{~min}$. The reaction was quenched with $\mathrm{MeOH}(20 \mathrm{~mL})$ and aqueous solution of $\mathrm{NH}_{4} \mathrm{Cl}(20 \mathrm{~mL})$, and extracted with EtOAc $(3 \times 20 \mathrm{~mL})$. The organic layer was washed with brine, dried over anhydrous $\mathrm{MgSO}_{4}$, and concentrated under reduced pressure to give an orange oil. The compounds were purified by flash column chromatography on silica gel $\left(\mathrm{CH}_{2} \mathrm{Cl}_{2}\right.$ /pentane $1: 1$ to $\mathrm{CH}_{2} \mathrm{Cl}_{2}$ to $\mathrm{CH}_{2} \mathrm{Cl}_{2} / \mathrm{MeOH}$ 9:1) affording the corresponding 3-hydroxy-3-arylindolin-2ones.

A mixture of the corresponding 3-hydroxy-3-arylindolin-2-one (17.0 $\mathrm{mmol}$ ) in $95 \mathrm{~mL}$ of $\mathrm{HOAc}$, and $\mathrm{SnCl}_{2}$ (34.0 mmol, 2 equiv) was stirred at $110^{\circ} \mathrm{C}$ for $2 \mathrm{~h}$ and then cooled to room temperature. After elimination of the HOAc under vacuum, the residue was diluted with EtOAc. The solution was successively washed with water, an aqueous solution of $\mathrm{NaHCO}_{3}$, brine, and dried over $\mathrm{MgSO}_{4}$. The mixture was purified by flash chromatography on silica gel (Hexane/EtOAc 8:1 to 4:1) to give the final compounds as white solids. 
1-Benzyl-5-fluoro-3-phenylindolin-2-one (5f). Compound $\mathbf{5 f}$ was obtained according to the general procedure using 1-benzyl-5fluoroindoline-2,3-dione ${ }^{[36]}(4.34 \mathrm{~g}, 17.0 \mathrm{mmol})$. Purification by flash column chromatography on silica gel (Hexane/EtOAc: 8:1 to 4:1) afforded the pure compound as a white solid $(3.08 \mathrm{~g}, 9.7 \mathrm{mmol}, 57 \%)$; m.p. 118$119^{\circ} \mathrm{C} .{ }^{1} \mathrm{H}$ NMR $\left(500 \mathrm{MHz}, \mathrm{CDCl}_{3}\right) \delta=7.40-7.25(\mathrm{~m}, 8 \mathrm{H}), 7.24-7.19(\mathrm{~m}$, 2H), 6.93-6.87 (m, 2H), 6.73- $6.66(\mathrm{~m}, 1 \mathrm{H}), 4.99(\mathrm{~d}, J=15.7 \mathrm{~Hz}, 1 \mathrm{H})$, 4.89 (d, $J=15.7 \mathrm{~Hz}, 1 \mathrm{H}), 4.70(\mathrm{~s}, 1 \mathrm{H}) \mathrm{ppm} .{ }^{13} \mathrm{C} \mathrm{NMR}\left(126 \mathrm{MHz}, \mathrm{CDCl}_{3}\right)$ $\delta=175.7,159.31\left(\mathrm{~d},{ }^{1} \mathrm{~J} \mathrm{C}-\mathrm{F}=241.0 \mathrm{~Hz}\right), 139.4,136.1,135.6,130.5(\mathrm{~d}$, $\left.{ }^{3} J_{C-F}=8.5 \mathrm{~Hz}\right), 129.1,128.9,128.4,127.9,127.8,127.3,114.64\left(\mathrm{~d},{ }^{2} \mathrm{~J}_{\mathrm{C}-\mathrm{F}}\right.$ $=23.4 \mathrm{~Hz}), 113.16\left(\mathrm{~d},{ }^{2} \mathrm{~J}_{\mathrm{C}-\mathrm{F}}=24.8 \mathrm{~Hz}\right), 109.7\left(\mathrm{~d},{ }^{3} \mathrm{~J}_{\mathrm{C}-\mathrm{F}}=8.1 \mathrm{~Hz}\right), 52.3$, $44.1 \mathrm{ppm}$. IR (ATR): $\tilde{v}=3065,3031,2919,2869,1709,1614,1489$, 1456, 799, 741, 695, $649 \mathrm{~cm}^{-1}$. HRMS (ESI-QTOF) m/z: $[M+\mathrm{Na}]^{+}$Calcd. for $\mathrm{C}_{21} \mathrm{H}_{16} \mathrm{FNNaO} 340.1108$; Found 340.1110 .

1-Benzyl-5-chloro-3-phenylindolin-2-one $(\mathbf{5 g})$. Compound $\mathbf{5 g}$ was obtained according to the general procedure using 1-benzyl-5chloroindoline-2,3-dione ${ }^{[37]}(4.62 \mathrm{~g}, 17.0 \mathrm{mmol})$. Purification by flash column chromatography on silica-gel (Hexane/EtOAc: $8: 1$ to $4: 1$ ) afforded the pure compound as white solid $(2.27 \mathrm{~g}, 6.8 \mathrm{mmol}, 40 \%)$; m.p. 149-150 ${ }^{\circ} \mathrm{C} .{ }^{1} \mathrm{H}$ NMR $\left(500 \mathrm{MHz}, \mathrm{CDCl}_{3}\right) \delta=7.39-7.27(\mathrm{~m}, 8 \mathrm{H}), 7.22-7.19$ (m, 2H), 7.17 (ddd, $J=8.3,2.1,0.8 \mathrm{~Hz}, 1 \mathrm{H}), 7.15-7.13(\mathrm{~m}, 1 \mathrm{H}), 6.69(\mathrm{~d}, J$ $=8.3 \mathrm{~Hz}, 1 \mathrm{H}), 4.99(\mathrm{~d}, J=15.6 \mathrm{~Hz}, 1 \mathrm{H}), 4.89(\mathrm{~d}, J=15.6 \mathrm{~Hz}, 1 \mathrm{H}), 4.69(\mathrm{~s}$ $1 \mathrm{H}) \mathrm{ppm} .{ }^{13} \mathrm{C} \mathrm{NMR}\left(126 \mathrm{MHz}, \mathrm{CDCl}_{3}\right) \delta=175.6,142.1,136.0,135.5$, 130.6, 129.1, 128.9, 128.4, 128.3, 128.2, 127.9, 127.8, 127.3, 125.5, 110.1, 52.1, $44.1 \mathrm{ppm}$. IR (ATR): $\tilde{v}=3063,3034,2921,2871,1709$, 1604, 1487, 1453, 1428, 743, 697, 663, cm-1. HRMS (ESI-QTOF) m/z: $[M+N a]^{+}$Calcd. for $\mathrm{C}_{21} \mathrm{H}_{16} \mathrm{CINNaO} 356.0813$; Found 356.0814.

1-Benzyl-5-bromo-3-phenylindolin-2-one (5h). Compound $5 \mathrm{~h}$ - was obtained according to the general procedure using 1-benzyl-5bromoindoline-2,3-dione ${ }^{[38]}(5.37 \mathrm{~g}, 17.0 \mathrm{mmol})$. Purification by flash column chromatography on silica gel (Hexane/EtOAc: 8:1 to 4:1) afforded the pure compound as a white solid $(3.34 \mathrm{~g}, 8.8 \mathrm{mmol}, 52 \%)$; m.p. 173$174{ }^{\circ} \mathrm{C} .{ }^{1} \mathrm{H}$ NMR $\left(500 \mathrm{MHz}, \mathrm{CDCl}_{3}\right) \delta=7.39-7.27(\mathrm{~m}, 10 \mathrm{H}), 7.20(\mathrm{~m}, 2 \mathrm{H})$ $6.65(\mathrm{~d}, J=8.3 \mathrm{~Hz}, 1 \mathrm{H}), 4.98(\mathrm{~d}, J=15.6 \mathrm{~Hz}, 1 \mathrm{H}), 4.88(\mathrm{~d}, J=15.7 \mathrm{~Hz}$, $1 \mathrm{H}), 4.70(\mathrm{~s}, 1 \mathrm{H}) \mathrm{ppm} .{ }^{13} \mathrm{C} \mathrm{NMR}\left(126 \mathrm{MHz}, \mathrm{CDCl}_{3}\right) \delta=175.5,142.6$, 136.0, 135.4, 131.2, 131.0, 129.1, 128.9, 128.4, 128.2, 127.9, 127.8, 127.3, 115.5, 110.7, 52.0, $44.0 \mathrm{ppm}$. IR (ATR): $\tilde{v}=3059,3034,2921$, $2871,1709,1600,1483,1453,713,697 \mathrm{~cm}^{-1}$. HRMS (ESI-QTOF) m/z: $[M+N a]^{+}$Calcd. for $\mathrm{C}_{21} \mathrm{H}_{16} \mathrm{BrNNaO} 400.0307$; Found 400.0316 .

1-Benzyl-5-methoxy-3-phenylindolin-2-one (5i). Compound $5 \mathbf{i}$ was obtained according to the general procedure using 1-benzyl-5methoxyindoline-2,3-dione ${ }^{[37]}(4.54 \mathrm{~g}, 17.0 \mathrm{mmol})$. Purification by flash column chromatography on silica gel (Hexane/EtOAc: 8:1 to 4:1) afforded the pure compound as a white solid $(4.20 \mathrm{~g}, 12.75 \mathrm{mmol}, 75 \%)$; m.p. $127-128{ }^{\circ} \mathrm{C} . \quad{ }^{1} \mathrm{H} \mathrm{NMR}\left(400 \mathrm{MHz}, \mathrm{CDCl}_{3}\right) \delta=7.38-7.19(\mathrm{~m}, 10 \mathrm{H}), 6.78-$ $6.75(\mathrm{~m}, 1 \mathrm{H}), 6.74-6.69(\mathrm{~m}, 1 \mathrm{H}), 6.65(\mathrm{~d}, J=8.5 \mathrm{~Hz}, 1 \mathrm{H}), 4.96(\mathrm{~d}, J=$ $16.0 \mathrm{~Hz}, 1 \mathrm{H}$ ), $4.86(\mathrm{~d}, J=15.7 \mathrm{~Hz}, 1 \mathrm{H}), 4.67$ (s, 1H), 3.70 (s, 3H) ppm. ${ }^{13} \mathrm{C} \mathrm{NMR}\left(126 \mathrm{MHz}, \mathrm{CDCl}_{3}\right) \delta=175.8,156.1,137.0,136.8,136.0,130.2$, 129.0, 128.8, 128.5, 127.6, 127.6, 127.3, 112.9, 112.3, 109.6, 55.7, 52.5, $44.0 \mathrm{ppm}$. IR (ATR): $\tilde{v}=3063,3034,2917,2833,1700,1600,1491,793$, 734, $697 \mathrm{~cm}^{-1}$. HRMS (ESI-QTOF) m/z: $[\mathrm{M}+\mathrm{Na}]^{+}$Calcd. for $\mathrm{C}_{22} \mathrm{H}_{19} \mathrm{NNaO}_{2}$ 352.1308; Found 352.1313.

1-Benzyl-3-phenyl-7-(trifluoromethyl)indolin-2-one (5j). Compound $\mathbf{5 j}$ was obtained according to the general procedure using 1-benzyl-7- (trifluoromethyl)indoline-2,3-dione ${ }^{[39]}(5.19 \mathrm{~g}, 17.0 \mathrm{mmol})$. Purification by flash column chromatography on silica gel (Hexane/EtOAc: $8: 1$ to $4: 1$ ) afforded the pure compound as a white solid $(2.19 \mathrm{~g}, 5.95 \mathrm{mmol}, 35 \%)$; m.p. $178-179^{\circ} \mathrm{C} .{ }^{1} \mathrm{H} \mathrm{NMR}\left(500 \mathrm{MHz}, \mathrm{CDCl}_{3}\right) \delta=7.60(\mathrm{~d}, J=8.1 \mathrm{~Hz}, 1 \mathrm{H})$, 7.40-7.30 (m, 4H), 7.29-7.24 (m, 2H), 7.23-7.18 (m, 3H), 7.16-7.10 (m, $3 \mathrm{H}), 5.28(\mathrm{~d}, J=16.9 \mathrm{~Hz}, 1 \mathrm{H}), 5.20(\mathrm{~d}, J=16.9 \mathrm{~Hz}, 1 \mathrm{H}), 4.72(\mathrm{~s}, 1 \mathrm{H})$ ppm. ${ }^{13} \mathrm{C}$ NMR $\left(126 \mathrm{MHz}, \mathrm{CDCl}_{3}\right) \delta=177.0,141.7,136.3,136.0,131.6$, 129.1, 129.0, 128.5, 128.3, 128.0, 126.9, 126.6 (q, $\left.{ }^{3} \mathrm{JC}-\mathrm{F}=6.2 \mathrm{~Hz}\right) 125.8$, 123.4 (q, $\left.{ }^{1} \mathrm{JC}-\mathrm{F}=271.7 \mathrm{~Hz}\right), 122.2,112.9\left(\mathrm{q},{ }^{2} \mathrm{JC}-\mathrm{F}=32.0 \mathrm{~Hz}\right), 50.7,45.6$ ppm. IR (ATR): $\tilde{v}=3038,2925,1725,1591,1495,1453,697 \mathrm{~cm}^{-1}$. HRMS (ESI-QTOF) m/z: [M+Na] $]^{+}$Calcd. for $\mathrm{C}_{22} \mathrm{H}_{16} \mathrm{~F}_{3} \mathrm{NNaO} 390.1076$; Found 390.1083 .

1-Benzyl-3-(4-(trifluoromethyl)phenyl)indolin-2-one (5k). Compound $\mathbf{5 k}$ was obtained according to the general procedure using 1 benzylindoline-2,3-dione ${ }^{[40]}(4.03 \mathrm{~g}, 17.0 \mathrm{mmol})$. Purification by flash column chromatography on silica gel (Hexane/EtOAc: 8:1 to 4:1) afforded the pure compound as a white solid ( $3.25 \mathrm{~g}, 8.84 \mathrm{mmol}, 52 \%)$; m.p. 145$146{ }^{\circ} \mathrm{C}$. ${ }^{1} \mathrm{H}$ NMR $\left(500 \mathrm{MHz}, \mathrm{CDCl}_{3}\right) \delta=7.61(\mathrm{~d}, J=8.1 \mathrm{~Hz}, 2 \mathrm{H}), 7.36(\mathrm{~d}$, $J=8.4 \mathrm{~Hz}, 2 \mathrm{H}), 7.33-7.23(\mathrm{~m}, 5 \mathrm{H}), 7.24(\mathrm{dt}, J=7.9,1.0 \mathrm{~Hz}, 1 \mathrm{H}), 7.15(\mathrm{~m}$, $1 \mathrm{H}$ ), 7.05 (td, $J=7.6,0.9 \mathrm{~Hz}, 1 \mathrm{H}$ ), 6.83 (ddd, $J=7.8,1.0,0.5 \mathrm{~Hz}, 1 \mathrm{H}$ ), $4.99(\mathrm{~d}, J=15.6 \mathrm{~Hz}, 1 \mathrm{H}), 4.90(\mathrm{~d}, J=15.6 \mathrm{~Hz}, 1 \mathrm{H}), 4.77(\mathrm{~s}, 1 \mathrm{H}) \mathrm{ppm} .{ }^{13} \mathrm{C}$ $\operatorname{NMR}\left(126 \mathrm{MHz}, \mathrm{CDCl}_{3}\right) \delta=175.2,143.6,140.7,135.7,129.9$ (q, ${ }^{2} \mathrm{~J}_{\mathrm{C}-\mathrm{F}}=$ $32.5 \mathrm{~Hz}$ ), 128.9, 128.9, 128.8, 127.9, 127.8, 127.4, 125.9 (q, ${ }^{3} \mathrm{~J}_{\mathrm{C}-\mathrm{F}}=3.7$ $\mathrm{Hz}), 124.0\left(\mathrm{q},{ }^{1} \mathrm{~J}_{\mathrm{C}-\mathrm{F}}=273.8 \mathrm{~Hz}\right), 109.5,51.7,44.1 \mathrm{ppm}$. IR (ATR): $\tilde{v}=$ 3038, 2925, 1704, 1608, 1491, 1466, 734, $697 \mathrm{~cm}^{-1}$. HRMS (ESI-QTOF) $\mathrm{m} / \mathrm{z}:[\mathrm{M}+\mathrm{Na}]^{+}$Calcd. for $\mathrm{C}_{22} \mathrm{H}_{16} \mathrm{~F}_{3} \mathrm{NNaO} 390.1076$; Found 390.1080.

General procedure for the enantioselective Michael addition. A mixture of oxindole $5 \mathbf{a}-\mathbf{n}(0.15 \mathrm{mmol})$, catalyst $(0.0075 \mathrm{mmol}, 0.05$ equiv) and nitroolefins 6 a-f $(0.3 \mathrm{mmol}, 2$ equiv) in $0.2 \mathrm{~mL}$ of DCM was stirred at r.t. in Wheaton vial until consumption of the starting material (monitored by ${ }^{1} \mathrm{H}$-NMR). After solvent removal under reduced pressure, the crude mixture was purified by flash column chromatography to afford the corresponding product. The enantiomeric excess was determined by chiral-phase HPLC analysis using mixtures of hexane/isopropanol as eluent.

(R)-1-Methyl-3-((R)-2-nitro-1-phenylethyl)-3-phenylindolin-2-one

(7aa). Product $7 \mathrm{aa}$ was obtained according to the general procedure, using oxindole $\mathbf{5 a}$, nitrostyrene $\mathbf{6 a}$ and catalyst Th-sq-la at $-78^{\circ} \mathrm{C}$. The crude reaction mixture was purified by flash chromatography (Hexane/EtOAc: 8:1 to 4:1) leading to compound 7aa as an inseparable mixture of diastereoisomers. White solid (55 mg, $0.147 \mathrm{mmol}, 98 \%)$. [ $\left.\alpha_{D}^{23}\right]$ $=+130.5\left[\left(c=1.00, \mathrm{CHCl}_{3}\right)(\mathrm{dr}=93: 7,96 \%\right.$ ee for the major diastereomer)]. ${ }^{1} \mathrm{H}$ NMR for major diastereomer $\left(500 \mathrm{MHz}, \mathrm{CDCl}_{3}\right) \delta=$ $7.70(\mathrm{dt}, J=8.3,1.1 \mathrm{~Hz}, 2 \mathrm{H}), 7.48-7.38(\mathrm{~m}, 4 \mathrm{H}), 7.29-7.23(\mathrm{~m}, 1 \mathrm{H}), 7.14-$ $7.08(\mathrm{~m}, 1 \mathrm{H}), 7.07-7.01(\mathrm{~m}, 3 \mathrm{H}), 6.84-6.79(\mathrm{~m}, 2 \mathrm{H}), 6.64(\mathrm{dd}, J=8.3,1.1$ $\mathrm{Hz}, 1 \mathrm{H}$ ), 5.00 (dd, $J=12.8,12.1 \mathrm{~Hz}, 1 \mathrm{H}$ ), $4.83(\mathrm{dd}, J=15.3,12.1 \mathrm{~Hz}, 1 \mathrm{H}$ ), $4.74\left(\mathrm{dd}, J=16.0,12.7 \mathrm{~Hz}, 1 \mathrm{H}\right.$ ), 2.70 (s, 3H) ppm. ${ }^{13} \mathrm{C}$ NMR for major diastereomer $\left(101 \mathrm{MHz}, \mathrm{CDCl}_{3}\right) \delta=175.3,144.6,135.6,133.4,129.4$, 129.2, 128.7, 128.4, 128.1, 127.7, 127.7, 126.7, 126.1, 122.3, 108.8, $76.0,59.7,50.7,25.9 \mathrm{ppm}$. IR (ATR): $\tilde{v}=3060,2923,1709,1610,1552$, 1493, $699 \mathrm{~cm}^{-1}$. HPLC: Lux-Amylose-1 column, hexane/iso-propanol $=$ 90:10, $1.0 \mathrm{~mL} / \mathrm{min}, \lambda=254 \mathrm{~nm}$. Dr 93:7. Major diastereoisomer: $t_{r}=$ $14.72 \mathrm{~min}$ (minor enantiomer), $t_{r}=17.66 \mathrm{~min}$ (major enantiomer) (er 98:2). Minor diastereoisomer: $t_{r}=12.17 \mathrm{~min}$ (major enantiomer), $t_{r}=16.67 \mathrm{~min}$ 
(minor enantiomer) (er 77:23). HRMS (ESI-QTOF) m/z: [M+Na] ${ }^{+}$Calcd for $\mathrm{C}_{23} \mathrm{H}_{20} \mathrm{~N}_{2} \mathrm{NaO}_{3}$ 395.1366; Found 395.1384.

\section{(R)-1-Benzyl-3-((R)-2-nitro-1-phenylethyl)-3-phenylindolin-2-one}

(7ba). Product $7 \mathbf{b a}$ was obtained from oxindole $5 \mathrm{~b}$, nitrostyrene $\mathbf{6 a}$ and catalyst Th-sq-Ilb. The crude reaction mixture was purified by flash chromatography (Hexane/EtOAc: 8:1 to 4:1) leading to compound 7ba as an inseparable mixture of diastereoisomers. White solid, $(63 \mathrm{mg}, 0.142$ mmol, 98\%). $\left[\alpha_{D}^{23}\right]=+86.5\left[\left(c=1.00, \mathrm{CHCl}_{3}\right)(\mathrm{dr}=94: 6,98 \%\right.$ ee for the major diastereomer)]. ${ }^{1} \mathrm{H}$ NMR for major diastereomer $\left(500 \mathrm{MHz}, \mathrm{CDCl}_{3}\right)$ $\delta=7.74-7.69(\mathrm{~m}, 2 \mathrm{H}), 7.48-7.41(\mathrm{~m}, 3 \mathrm{H}), 7.30-7.21(\mathrm{~m}, 4 \mathrm{H}), 7.18-7.04$ (m, 5H), 6.97-6.92 (m, 2H), 6.53-6.47 (m, 1H), $6.42(\mathrm{dd}, J=7.8,1.4 \mathrm{~Hz}$, 2H), $5.06(\mathrm{dd}, J=12.4,12.4 \mathrm{~Hz}, 1 \mathrm{H}), 4.96(\mathrm{dd}, J=15.1,12.2 \mathrm{~Hz}, 1 \mathrm{H}$ ), $4.80(\mathrm{~d}, J=15.7 \mathrm{~Hz}, 1 \mathrm{H}), 4.77(\mathrm{dd}, J=15.4,12.5 \mathrm{~Hz}, 1 \mathrm{H}), 4.28(\mathrm{~d}, J=$ 16.1 Hz, 1H) ppm. ${ }^{13} \mathrm{C}$ NMR for major diastereomer $\left(101 \mathrm{MHz}, \mathrm{CDCl}_{3}\right) \delta$ $=175.5,143.8,136.3,134.7,134.0,129.4,129.4,129.3,128.6,128.5$, 128.4, 128.3, 127.6, 127.3, 127.2, 126.4, 126.2, 122.4, 110.4, 76.8, 59.6, 50.7, 43.9 ppm. IR (ATR): $v^{=}=3065,2923,1710,1610,1552,1489,695$ $\mathrm{cm}^{-1}$. HPLC: Lux-i-Amylose-1 column, hexane/iso-propanol $=90: 10,1.0$ $\mathrm{mL} / \mathrm{min}, \lambda=254 \mathrm{~nm}$. Dr 94:6. Major diastereoisomer: $t_{r}=20.42 \mathrm{~min}$ (minor enantiomer), $t_{r}=48.82 \mathrm{~min}$ (major enantiomer) (er 99:1). Minor diastereoisomer: $t_{r}=26.10 \mathrm{~min}$ (major enantiomer), $t_{r}=28.92 \mathrm{~min}$ (minor enantiomer) (er 80:20). HRMS (ESI-QTOF) $\mathrm{m} / \mathrm{z}:[\mathrm{M}+\mathrm{Na}]^{+}$Calcd. for $\mathrm{C}_{29} \mathrm{H}_{24} \mathrm{~N}_{2} \mathrm{NaO}_{3}$ 471.1679; Found 471.1685.

tert-Butyl (R)-3-((R)-2-nitro-1-phenylethyl)-2-oxo-3-phenylindoline-1carboxylate (7ca). ${ }^{[15],[18]}$ Product 7ca was obtained according to the general procedure, from oxindole $5 \mathrm{c}$, nitrostyrene $\mathbf{6 a}$ and catalyst Th-sqla. The crude reaction mixture was purified by flash chromatography (Hexane/EtOAc: 8:1 to 4:1) leading to compound 7ca as an inseparable mixture of diastereoisomers. Colorless oil $(46 \mathrm{mg}, 0.10 \mathrm{mmol}, 67 \%$ ). $[\alpha]_{D}{ }^{23}=+36.6\left(c=1.00, \mathrm{CHCl}_{3}\right)[(\mathrm{dr}=71: 29,36 \%$ ee for the major diastereomer)]. Lit. ${ }^{15}[\alpha]_{D^{23}}=+242.7\left(c=0.5, \mathrm{CHCl}_{3}\right)(\mathrm{dr} 98: 2$, ee $89 \%$ for $(R, R)$-enantiomer). ${ }^{1} \mathrm{H} \mathrm{NMR}$ for major diastereomer $\left(400 \mathrm{MHz}, \mathrm{CDCl}_{3}\right)$ $\delta=7.69(\mathrm{dt}, J=7.7,1.3 \mathrm{~Hz}, 1 \mathrm{H}), 7.59(\mathrm{ddq}, J=9.5,8.4,1.3 \mathrm{~Hz}, 3 \mathrm{H})$, 7.45-7.38 (m, 4H), 7.16-7.11 (m, 1H), 7.06-7.01 (m, 3H), 6.77 (dt, $J=6.9$, $1.2 \mathrm{~Hz}, 2 \mathrm{H}), 4.94(\mathrm{t}, J=12.6,12.2 \mathrm{~Hz}, 1 \mathrm{H}), 4.87(\mathrm{dd}, J=12.1,3.1 \mathrm{~Hz}$, $1 \mathrm{H}), 4.77(\mathrm{dd}, J=12.7,3.1 \mathrm{~Hz}, 1 \mathrm{H}), 1.43(\mathrm{~s}, 9 \mathrm{H}) \mathrm{ppm} .{ }^{13} \mathrm{C}$ NMR for major diastereomer $\left(101 \mathrm{MHz}, \mathrm{CDCl}_{3}\right) \delta=173.9,148.3,141.0,135.4,132.8$, 129.6, 129.3, 128.8, 128.6, 128.4, 128.0, 127.9, 126.6, 125.7, 124.2, 115.7, 84.2, 75.8, 60.1, 50.8, 27.8 ppm. IR (ATR): $\tilde{v}=3973,1784,1709$, 1610, 1551, 1468, 741, $695 \mathrm{~cm}^{-1}$. HPLC: Lux-Amylose-1 column, hexane/iso-propanol $=98: 2,0.5 \mathrm{~mL} / \mathrm{min}, \lambda=254 \mathrm{~nm}$. Dr 71:29. Major diastereoisomer: $t_{r}=26.47 \mathrm{~min}$ (minor enantiomer), $t_{r}=36.19 \mathrm{~min}$ (major enantiomer) (er 68:32). Minor diastereoisomer: $t_{r}=25.22 \mathrm{~min}$ (major enantiomer), $t_{r}=60.22 \mathrm{~min}$ (minor enantiomer) (er 58:42). HRMS (ESIQTOF) $\mathrm{m} / \mathrm{z}:[\mathrm{M}+\mathrm{Na}]^{+}$Calcd. for $\mathrm{C}_{27} \mathrm{H}_{26} \mathrm{~N}_{2} \mathrm{NaO}_{5}$ 481.1734; Found 481.1752 .

$(R)$-3-((R)-2-Nitro-1-phenylethyl)-3-phenylindolin-2-one $\quad$ (7da). ${ }^{[16]}$ Product 7da was obtained according to the general procedure, by reacting oxindole $\mathbf{5 d}$, nitrostyrene $\mathbf{6 a}$ and catalyst Th-sq-la. The crude reaction mixture was purified by flash chromatography (Hexane/EtOAc: $8: 1$ to $4: 1$ ) leading to compound $7 \mathrm{da}$ as an inseparable mixture of diastereoisomers. Colorless oil (52 mg, $0.144 \mathrm{mmol}, 96 \%) \cdot[\alpha]_{\mathrm{D}}{ }^{23}=+$ $137.7\left[\left(c=0.70, \mathrm{CHCl}_{3}\right)(\mathrm{dr}=81: 19,78 \%\right.$ ee for the major diastereomer $\left.)\right]$ ${ }^{1} \mathrm{H}$ NMR for major diastereomer $\left(500 \mathrm{MHz}, \mathrm{CDCl}_{3}\right) \delta=7.98(\mathrm{br}, 1 \mathrm{H})$,
7.69-7.62 (m, 2H), 7.43-7.39 (m, 4H), 7.28-7.22 (m, 1H), 7.12-7.08 (m, $1 \mathrm{H}), 7.04-6.99(\mathrm{~m}, 3 \mathrm{H}), 6.86(\mathrm{dd}, J=8.3,1.3 \mathrm{~Hz}, 2 \mathrm{H}), 6.75(\mathrm{dt}, J=7.8$, $0.8 \mathrm{~Hz}, 1 \mathrm{H}), 5.00(\mathrm{t}, J=12.8,12.0 \mathrm{~Hz}, 1 \mathrm{H}), 4.83(\mathrm{dd}, J=15.2,12.1 \mathrm{~Hz}$, $1 \mathrm{H}), 4.74(\mathrm{dd}, J=15.9,12.7 \mathrm{~Hz}, 1 \mathrm{H}) \mathrm{ppm} .{ }^{13} \mathrm{C} N M R$ for major diastereomer $\left(101 \mathrm{MHz}, \mathrm{CDCl}_{3}\right) \delta=177.5,141.7,135.7,133.5,129.5$, 129.2, 128.9, 128.4, 128.2, 128.0, 127.8, 127.6, 126.4, 122.4, 110.7, 76.3, 60.0, 50.4 ppm. IR (ATR): $\tilde{v}=3210,2923,1709,1618,1552,1473$, 737, $695 \mathrm{~cm}^{-1}$. HPLC: Chiralpak IA column, hexane/iso-propanol = 95:5, $1.0 \mathrm{~mL} / \mathrm{min}, \lambda=254 \mathrm{~nm}$. $\operatorname{Dr}$ 81:19. Major diastereoisomer: $t_{r}=29.99 \mathrm{~min}$ (major enantiomer), $t_{r}=37.08 \mathrm{~min}$ (minor enantiomer) (er 89:11). Minor diastereoisomer: $t_{r}=32.50 \mathrm{~min}$ (major enantiomer), $t_{r}=34.73 \mathrm{~min}$ (minor enantiomer). (er 68:32). HRMS (ESI-QTOF) $\mathrm{m} / \mathrm{z}$ : $[\mathrm{M}+\mathrm{Na}]^{+}$Calcd. for $\mathrm{C}_{22} \mathrm{H}_{18} \mathrm{~N}_{2} \mathrm{NaO}_{3} 381.1210$; Found 381.1219.

(R)-1-Benzyl-5-methyl-3-((R)-2-nitro-1-phenylethyl)-3-phenylindolin2-one (7ea). Product 7ea was obtained according to the general procedure, using oxindole $5 \mathrm{e}$, nitrostyrene $\mathbf{6 a}$ and catalyst Th-sq-llb. The crude reaction mixture was purified by flash chromatography (Hexane/EtOAc: 8:1 to 4:1) leading to compound 7ea as an inseparable

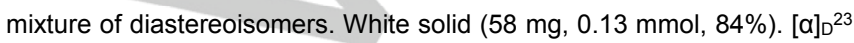
$=+31.0\left[\left(c=0.10, \mathrm{CHCl}_{3}\right)(\mathrm{dr}=89: 11,96 \%\right.$ ee for the major diastereomer)]. ${ }^{1} \mathrm{H}$ NMR for major diastereomer $\left(500 \mathrm{MHz}, \mathrm{CDCl}_{3}\right) \delta=$ 7.73-7.70 (m, 2H), $7.44(\mathrm{dd}, J=8.5,6.8 \mathrm{~Hz}, 2 \mathrm{H}), 7.39(\mathrm{~d}, J=7.3 \mathrm{~Hz}, 1 \mathrm{H})$, 7.27-7.22 (m, 2H), $7.12(\mathrm{td}, J=7.6,2.2 \mathrm{~Hz}, 3 \mathrm{H}), 7.09-7.04(\mathrm{~m}, 3 \mathrm{H}), 6.98-$ $6.92(\mathrm{~m}, 2 \mathrm{H}), 6.41$ (dd, $J=7.3,1.7 \mathrm{~Hz}, 2 \mathrm{H}), 6.38(\mathrm{~d}, J=8.0 \mathrm{~Hz}, 1 \mathrm{H}), 5.07$ (dd, $J=12.6,12.1 \mathrm{~Hz}, 1 \mathrm{H}), 4.96(\mathrm{dd}, J=15.0,12.1,1 \mathrm{H}), 4.78(\mathrm{~d}, J=$ $15.5 \mathrm{~Hz}, 1 \mathrm{H}), 4.78(\mathrm{dd}, J=15.1,12.6 \mathrm{~Hz}, 1 \mathrm{H}), 4.26(\mathrm{~d}, J=16.1 \mathrm{~Hz}, 1 \mathrm{H})$, $2.48(\mathrm{~s}, 3 \mathrm{H}) \mathrm{ppm} .{ }^{13} \mathrm{C}$ NMR for major diastereomer $\left(126 \mathrm{MHz}, \mathrm{CDCl}_{3}\right) \delta=$ $175.4,141.5,136.5,134.8,134.1,132.0,129.8,129.4,129.2,128.5$, 128.4, 128.3, 128.3, 127.6, 127.3, 127.1, 126.8, 126.4, 110.1, 76.9, 59.7, 50.5, 43.9, 21.4 ppm. IR (ATR): $\tilde{v}=2923,1705,1601,1552,1497,728$, $695 \mathrm{~cm}^{-1}$. HPLC: Lux-i-Amylose-1 column, hexane/iso-propanol = 95:5, $1.0 \mathrm{~mL} / \mathrm{min}, \lambda=254 \mathrm{~nm}$. Dr 89:11. Major diastereoisomer: $t_{r}=22.79 \mathrm{~min}$ (minor enantiomer), $t_{r}=68.45 \mathrm{~min}$ (major enantiomer) (er 98:2). Minor diastereoisomer: $t_{r}=31.77 \mathrm{~min}$ (minor enantiomer), $t_{r}=34.18 \mathrm{~min}$ (major enantiomer) (er 86:14). HRMS (ESI-QTOF) m/z: $[M+N a]^{+}$Calcd. for $\mathrm{C}_{30} \mathrm{H}_{26} \mathrm{~N}_{2} \mathrm{NaO}_{3}$ 485.1836; Found 485.1844.

(R)-1-Benzyl-5-fluoro-3-((R)-2-nitro-1-phenylethyl)-3-phenylindolin-2one (7fa). Product $7 \mathrm{fa}$ was obtained according to the general procedure, using oxindole $5 f$, nitrostyrene $6 \mathrm{a}$ and catalyst Th-sq-Ilb at $-40^{\circ} \mathrm{C}$. The crude reaction mixture was purified by flash chromatography (Hexane/EtOAc: 8:1 to 4:1) leading to compound $7 \mathrm{fa}$ as an inseparable mixture of diastereoisomers. White solid $(66 \mathrm{mg}, 0.14 \mathrm{mmol}, 95 \%)$. [a] $]_{\mathrm{b}}^{23}$ $=+77.6\left[\left(c=1.00, \mathrm{CHCl}_{3}\right) \quad(\mathrm{dr}=92: 8,94 \%\right.$ ee for the major diastereomer)]. ${ }^{1} \mathrm{H}$ NMR for major diastereomer $\left(500 \mathrm{MHz}, \mathrm{CDCl}_{3}\right) \delta=$ 7.70-7.66 (m, 2H), 7.48-7.43 (m, 2H), 7.42-7.38 (m, 1H), 7.28-7.24 (m, $1 \mathrm{H}), 7.20(\mathrm{dd}, J=8.0,2.5 \mathrm{~Hz}, 1 \mathrm{H}), 7.16-7.11(\mathrm{~m}, 3 \mathrm{H}), 7.10-7.06(\mathrm{~m}, 2 \mathrm{H})$, 7.01-6.95 (m, 3H). 6.43-6.38 (m, 3H), $5.02(\mathrm{dd}, J=12.1,12.0,1 \mathrm{H}), 4.96$ $(\mathrm{dd}, J=14.5,12.0 \mathrm{~Hz}, 1 \mathrm{H}), 4.79(\mathrm{~d}, J=16.2 \mathrm{~Hz}, 1 \mathrm{H}), 4.75(\mathrm{dd}, J=14.3$, $12.0 \mathrm{~Hz}, 1 \mathrm{H}), 4.26(\mathrm{~d}, J=16.1 \mathrm{~Hz}, 1 \mathrm{H}) \mathrm{ppm} .{ }^{13} \mathrm{C}$ NMR for major diastereomer $\left(101 \mathrm{MHz}, \mathrm{CDCl}_{3}\right) \delta=175.3,158.7\left(\mathrm{~d},{ }^{1} \mathrm{~J}_{\mathrm{C}-\mathrm{F}}=242.1 \mathrm{~Hz}\right)$, 139.8, 135.7, 134.0 (d, J = 63.4 Hz), 129.4, 129.3, 128.8, 128.7, 128.7 $128.5,128.5,127.4,127.3,127.1,126.4,115.9\left(\mathrm{~d},{ }^{2} J_{C-F}=23.0 \mathrm{~Hz}\right)$, $114.1\left(\mathrm{~d},{ }^{2} \mathrm{JC}_{\mathrm{F}}=24.7 \mathrm{~Hz}\right), 111.0\left(\mathrm{~d},{ }^{3} \mathrm{JC}_{\mathrm{F}} \mathrm{F}=8.0 \mathrm{~Hz}\right), 76.4,60.1,50.5,44.0$ ppm. IR (ATR): $\tilde{v}=3060,2923,1705,1601,1552,1489,1452,737,695$ 
$\mathrm{cm}^{-1}$. HPLC: Lux-i-Amylose-1 column, hexane/iso-propanol $=95: 5,1.0$ $\mathrm{mL} / \mathrm{min}, \lambda=254 \mathrm{~nm}$. Dr 92:8. Major diastereoisomer: $t_{r}=27.45 \mathrm{~min}$ (minor enantiomer), $t_{r}=89.04 \mathrm{~min}$ (major enantiomer) (er 97:3). Minor diastereoisomer: $t_{r}=44.99 \mathrm{~min}$ (major enantiomer), $t_{r}=48.64 \mathrm{~min}$ (minor enantiomer) (er 67:32). HRMS (ESI-QTOF) $\mathrm{m} / \mathrm{z}$ : $[\mathrm{M}+\mathrm{Na}]^{+}$Calcd. for $\mathrm{C}_{29} \mathrm{H}_{23} \mathrm{FN}_{2} \mathrm{NaO}_{3}$ 489.1585; Found 489.1587

(R)-1-Benzyl-5-chloro-3-((R)-2-nitro-1-phenylethyl)-3-phenylindolin-

2-one (7ga). Product 7ga was obtained according to the general procedure, using oxindole $\mathbf{5 g}$, nitrostyrene $\mathbf{6 a}$ and catalyst Th-sq- $\mathbf{l l b}$ at $40^{\circ} \mathrm{C}$. The crude reaction mixture was purified by flash chromatography (Hexane/EtOAc: 8:1 to 4:1) leading to compound 7ga as an inseparable mixture of diastereoisomers. White solid (59 mg, $0.12 \mathrm{mmol}, 82 \%)$. [a] $]_{\mathrm{D}}^{23}$ $=+11.0\left[\left(c=0.40, \mathrm{CHCl}_{3}\right)(\mathrm{dr}=90: 10,94 \%\right.$ ee for the major diastereomer)]. ${ }^{1} \mathrm{H}$ NMR for major diastereomer $\left(500 \mathrm{MHz}, \mathrm{CDCl}_{3}\right) \delta=$ 7.71-7.63 (m, 2H), 7.50-7.43 (m, 2H), 7.43-7.39 (m, 2H), 7.30-7.24 (m, $2 \mathrm{H}), 7.18-7.11(\mathrm{~m}, 3 \mathrm{H}), 7.07(\mathrm{dd}, J=8.3,6.8 \mathrm{~Hz}, 2 \mathrm{H}), 7.01-6.94(\mathrm{~m}, 2 \mathrm{H})$, 6.44-6.35 (m, 3H), $5.03(\mathrm{t}, J=12.3,12.2 \mathrm{~Hz}, 1 \mathrm{H}), 4.95(\mathrm{dd}, J=14.9,12.1$ $\mathrm{Hz}, 1 \mathrm{H}), 4.78(\mathrm{~d}, J=16.1 \mathrm{~Hz}, 1 \mathrm{H}), 4.76(\mathrm{dd}, J=15.0,12.4 \mathrm{~Hz}, 1 \mathrm{H}), 4.26$ (d, $J=16.0 \mathrm{~Hz}, 1 \mathrm{H}$ ) ppm. ${ }^{13} \mathrm{C}$ NMR for major diastereomer $(101 \mathrm{MHz}$, $\left.\mathrm{CDCl}_{3}\right) \delta=175.1,142.4,135.6,134.2,133.6,129.8,129.4,129.3,129.2$, $128.7,128.7,128.5,128.5,127.9,127.4,127.4,127.0,126.4,111.3$, 76.4, 59.9, 50.5, 44.0 ppm. IR (ATR): $\tilde{v}=3061,2919,1709,1605,1552$, 1485, 737, $695 \mathrm{~cm}^{-1}$. HPLC: Lux-i-Amylose-1 column, hexane/isopropanol $=95: 5, \quad 1.0 \mathrm{~mL} / \mathrm{min}, \lambda=254 \mathrm{~nm}$. Dr 90:10. Major diastereoisomer: $t_{r}=23.42 \mathrm{~min}$ (minor enantiomer), $t_{r}=71.87 \mathrm{~min}$ (major enantiomer) (er 97:3). Minor diastereoisomer: $t_{r}=37.62 \mathrm{~min}$ (minor enantiomer), $t_{r}=40.14 \mathrm{~min}$ (major enantiomer) (er 71:29). HRMS (ESIQTOF) m/z: $[M+H]^{+}$Calcd. for $\mathrm{C}_{29} \mathrm{H}_{24} \mathrm{ClN}_{2} \mathrm{O}_{3}$ 483.1470; Found 483.1478.

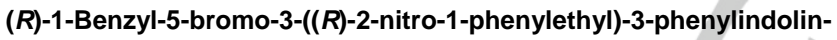
2-one (7ha). Product 7 ha was obtained according to the general procedure, using oxindole $5 \mathrm{~h}(1.5 \mathrm{mmol})$, nitrostyrene $\mathbf{6 a}(3.0 \mathrm{mmol})$ and catalyst Th-sq-Ilb $(0.075 \mathrm{mmol})$. The crude reaction mixture was purified by flash chromatography (Hexane/EtOAc: $8: 1$ to $4: 1$ ) and recrystallized from hexane-ethyl acetate leading to compound 7 ha as a mixture of diastereoisomers. White solid (568 mg, $1.08 \mathrm{mmol}, 72 \%$ ). $[\alpha]_{\mathrm{D}}{ }^{23}=+26.6$ $\left[\left(c=1.0, \mathrm{CHCl}_{3}\right)(\mathrm{dr}=97: 3,94 \%\right.$ ee for the major diastereomer $\left.)\right] .{ }^{1} \mathrm{H}$ NMR for major diastereomer $\left(500 \mathrm{MHz}, \mathrm{CDCl}_{3}\right) \delta=7.72-7.64(\mathrm{~m}, 2 \mathrm{H})$, $7.54(\mathrm{~d}, J=1.9 \mathrm{~Hz}, 1 \mathrm{H}), 7.46$ (dd, $J=8.3,6.7 \mathrm{~Hz}, 2 \mathrm{H}), 7.38(\mathrm{dd}, J=8.3$, $1.9 \mathrm{~Hz}, 1 \mathrm{H}), 7.28-7.24(\mathrm{~m}, 2 \mathrm{H}), 7.14(\mathrm{td}, J=7.5,6.8,1.7 \mathrm{~Hz}, 3 \mathrm{H}), 7.07$ (dd, $J=8.2,6.8 \mathrm{~Hz}, 2 \mathrm{H}), 7.01-6.95(\mathrm{~m}, 2 \mathrm{H}), 6.42-6.37(\mathrm{~m}, 2 \mathrm{H}), 6.35(\mathrm{~d}, J$ $=8.4 \mathrm{~Hz}, 1 \mathrm{H}), 5.02(\mathrm{dd}, J=12.2,12.2 \mathrm{~Hz}, 1 \mathrm{H}), 4.95(\mathrm{dd}, J=14.9,12.3$ $\mathrm{Hz}, 1 \mathrm{H}$ ), 4.78 (d, $J=12.2 \mathrm{~Hz}, 1 \mathrm{H}$ ), 4.75 (dd, $J=14.9,12.3 \mathrm{~Hz}, 1 \mathrm{H}$ ), 4.26 (d, $J=12.1 \mathrm{~Hz}, 1 \mathrm{H}) \mathrm{ppm} .{ }^{13} \mathrm{C}$ NMR for major diastereomer $(126 \mathrm{MHz}$, $\left.\mathrm{CDCl}_{3}\right) \delta=175.0,142.9,135.6,134.1,133.6,132.4,129.5,129.3,129.1$, $128.8,128.7,128.6,128.5,127.4,127.4,127.0,126.4,115.1,111.8$, $76.4,59.8,50.5,44.0$ ppm. IR (ATR): $v=3060,2923,1714,1606,1552$, 1481, 737, $695 \mathrm{~cm}^{-1}$. HPLC: Lux-i-Amylose-1 column, hexane/isopropanol $=95: 5,1.0 \mathrm{~mL} / \mathrm{min}, \quad \lambda=254 \mathrm{~nm}$. Dr 97:3. Major diastereoisomer: $t_{r}=25.77 \mathrm{~min}$ (minor enantiomer), $t_{r}=82.82 \mathrm{~min}$ (major enantiomer) (er 97:3). Minor diastereoisomer: $t_{r}=39.92 \mathrm{~min}$ (minor enantiomer), $t_{r}=44.28 \mathrm{~min}$ (major enantiomer) (er 91:9). HRMS (ESIQTOF) m/z: $[M+N a]^{+}$Calcd. for $\mathrm{C}_{29} \mathrm{H}_{23} \mathrm{BrN}_{2} \mathrm{NaO}_{3}$ 549.0784; Found 549.0786

\section{(R)-1-Benzyl-5-methoxy-3-((R)-2-nitro-1-phenylethyl)-3-}

phenylindolin-2-one (7ia). Product 7ia was obtained according to the general procedure, using oxindole $\mathbf{5 i}$, nitrostyrene $\mathbf{6 a}$ and catalyst Th-sqIlb. The crude reaction mixture was purified by flash chromatography (Hexane/EtOAc: 8:1 to 4:1) leading to compound 7ia as an inseparable mixture of diastereoisomers. White solid (51 $\mathrm{mg}, 0.11 \mathrm{mmol}, 71 \%)$. [a] $]^{23}$ $=+70.8\left[\left(c=0.90, \mathrm{CHCl}_{3}\right)(\mathrm{dr}=97: 3,98 \%\right.$ ee for the major diastereomer)]. ${ }^{1} \mathrm{H}$ NMR for major diastereomer $\left(500 \mathrm{MHz}, \mathrm{CDCl}_{3}\right) \delta=$ 7.75-7.69 (m, 2H), 7.47-7.42 (m, 2H), 7.40-7.35 (m, 1H), 7.28-7.23 (m, $1 \mathrm{H}), 7.12$ (dd, $J=8.2,6.9 \mathrm{~Hz}, 3 \mathrm{H}), 7.09-7.05(\mathrm{~m}, 2 \mathrm{H}), 7.03(\mathrm{~d}, J=2.5 \mathrm{~Hz}$, $1 \mathrm{H}), 7.00-6.96(\mathrm{~m}, 2 \mathrm{H}), 6.78(\mathrm{dd}, J=8.6,2.5 \mathrm{~Hz}, 1 \mathrm{H}), 6.40(\mathrm{dd}, J=7.9$, $1.4 \mathrm{~Hz}, 2 \mathrm{H}), 6.38(\mathrm{~d}, J=8.6 \mathrm{~Hz}, 1 \mathrm{H}), 4.95(\mathrm{dd}, J=12.4,12.3 \mathrm{~Hz}, 1 \mathrm{H})$, $4.91(\mathrm{dd}, J=15.0,12.1 \mathrm{~Hz}, 1 \mathrm{H}), 4.78(\mathrm{~d}, J=16.1 \mathrm{~Hz}, 1 \mathrm{H}), 4.75(\mathrm{dd}, J=$ 15.3, $12.5 \mathrm{~Hz}, 1 \mathrm{H}$ ), 4.24 (d, $J=16.0,1 \mathrm{H}$ ), 3.87 (s, 3H) ppm. ${ }^{13} \mathrm{C}$ NMR for major diastereomer $\left(101 \mathrm{MHz}, \mathrm{CDCl}_{3}\right) \delta=175.2,155.6,137.3,136.3$, $134.8,134.0,129.4,129.3,128.6,128.6,128.5,128.4,128.3,127.6$, 127.1, 126.4, 114.3, 112.8, 110.6, 76.7, 60.0, 55.9, 50.5, 44.0 ppm. IR (ATR): $v=3065,2923,1705,1601,1556,1493,695 \mathrm{~cm}^{-1}$. HPLC: Lux-iAmylose-1 column, hexane/iso-propanol $=95: 5,1.0 \mathrm{~mL} / \mathrm{min}, \lambda=254 \mathrm{~nm}$. Dr 97:3. Major diastereoisomer: $t_{r}=48.98 \mathrm{~min}$ (minor enantiomer), $t_{r}=$ $165.11 \mathrm{~min}$ (major enantiomer) (er 99:1). Minor diastereoisomer: $t_{r}=$ $89.55 \mathrm{~min}$ (major enantiomer), $t_{r}=106.69 \mathrm{~min}$ (minor enantiomer) (er 86:14). HRMS (ESI-QTOF) m/z: $[M+N a]^{+}$Calcd. for $\mathrm{C}_{30} \mathrm{H}_{26} \mathrm{~N}_{2} \mathrm{NaO}_{4}$ 501.1785; Found 501.1783.

(R)-1-Benzyl-3-((R)-2-nitro-1-phenylethyl)-3-phenyl-7-

(trifluoromethyl)indolin-2-one (7ja). Product 7ja was obtained according to the general procedure, from oxindole $5 \mathbf{j}$, nitrostyrene $\mathbf{6 a}$ and catalyst Th-sq-Ilb at $-40^{\circ} \mathrm{C}$. The crude reaction mixture was purified by flash chromatography (Hexane/EtOAc: 8:1 to 4:1) leading to compound $7 \mathbf{j a}$ as an inseparable mixture of diastereoisomers. White solid $(76 \mathrm{mg}$, $0.147 \mathrm{mmol}, 98 \%) .[\alpha]_{\mathrm{D}}{ }^{23}=+71.0\left[\left(c=0.10, \mathrm{CHCl}_{3}\right)(\mathrm{dr}=81: 19,94 \%\right.$ ee for the major diastereomer)]. ${ }^{1} \mathrm{H}$ NMR for major diastereomer $(500 \mathrm{MHz}$, $\left.\mathrm{CDCl}_{3}\right) \delta=7.71(\mathrm{ddd}, J=8.2,3.4,1.3 \mathrm{~Hz}, 1 \mathrm{H}), 7.66-7.61(\mathrm{~m}, 2 \mathrm{H}), 7.60$ (dd, $J=7.6,1.3 \mathrm{~Hz}, 1 \mathrm{H}), 7.48-7.43(\mathrm{~m}, 2 \mathrm{H}), 7.30-7.24(\mathrm{~m}, 2 \mathrm{H}), 7.16-7.11$ $(\mathrm{m}, 2 \mathrm{H}), 7.11-7.06(\mathrm{~m}, 1 \mathrm{H}), 7.06-7.02(\mathrm{~m}, 3 \mathrm{H}), 6.89-6.84(\mathrm{~m}, 2 \mathrm{H}), 6.48-$ $6.42(\mathrm{~m}, 2 \mathrm{H}), 4.99(\mathrm{dd}, J=12.1,10.6 \mathrm{~Hz}, 1 \mathrm{H}), 4.97(\mathrm{dd}, J=11.5,10.6 \mathrm{~Hz}$, $1 \mathrm{H}), 4.81$ (dd, $J=12.1,11.5 \mathrm{~Hz}, 1 \mathrm{H}), 4.81$ (d, $J=17.6 \mathrm{~Hz}, 1 \mathrm{H}), 4.70$ (d, $J$ $=17.3 \mathrm{~Hz}, 1 \mathrm{H}) \mathrm{ppm} .{ }^{13} \mathrm{C}$ NMR for major diastereomer $\left(101 \mathrm{MHz}, \mathrm{CDCl}_{3}\right)$ $\delta=176.8,135.6,133.2,130.7,129.8,129.7,129.5,129.0,128.8,128.7$, $128.5,128.4,128.2,127.7$ (q, ${ }^{3} \mathrm{~J}_{\mathrm{C}-\mathrm{F}}=6.3 \mathrm{~Hz}$ ), 126.5, 126.5, 125.0, 122.9 (q, ${ }^{1} \mathrm{JC}_{\mathrm{C}-\mathrm{F}}=272.4 \mathrm{~Hz}$ ), 121.8, 113.7 (q, ${ }^{2} \mathrm{JC}_{\mathrm{C}-\mathrm{F}}=32.8 \mathrm{~Hz}$ ), 76.4, 58.2, 50.5, $45.8 \mathrm{ppm}$. IR (ATR): $\tilde{v}=\tilde{v}=2977,1734,1597,1556,1452,1443,733$, $695 \mathrm{~cm}^{-1}$. HPLC: Lux-i-Amylose-1 column, hexane/iso-propanol $=97: 3$, $1.0 \mathrm{~mL} / \mathrm{min}, \lambda=254 \mathrm{~nm}$. Dr 81:19. Major diastereoisomer: $t_{r}=27.19 \mathrm{~min}$ (minor enantiomer), $t_{r}=79.48 \mathrm{~min}$ (major enantiomer) (er 97:3). Minor diastereoisomer: $t_{r}=24.30 \mathrm{~min}$ (minor enantiomer), $t_{r}=25.51 \mathrm{~min}$ (major enantiomer) (er 67:33). HRMS (ESI-QTOF) $\mathrm{m} / \mathrm{z}$ : $[M+\mathrm{Na}]^{+}$Calcd. for $\mathrm{C}_{30} \mathrm{H}_{23} \mathrm{~F}_{3} \mathrm{~N}_{2} \mathrm{NaO}_{3}$ 539.1553; Found 539.1562.

(R)-1-Benzyl-3-((R)-2-nitro-1-phenylethyl)-3-(4-

(trifluoromethyl)phenyl)indolin-2-one (7ka). Product 7ka was obtained according to the general procedure, using oxindole $\mathbf{5 k}$, nitrostyrene $\mathbf{6 a}$ and catalyst Th-sq-Ilb. The crude reaction mixture was purified by flash chromatography (Hexane/Ethyl acetate: 8:1 to 4:1) leading to compound $7 \mathbf{k a}$ as an inseparable mixture of diastereoisomers. White solid $(76 \mathrm{mg}$, 
$0.147 \mathrm{mmol}, 98 \%) .[\alpha]_{\mathrm{D}}^{23}=+66.0\left[\left(c=0.10, \mathrm{CHCl}_{3}\right)(\mathrm{dr}=90: 10,96 \%\right.$ ee for the major diastereomer)]. ${ }^{1} \mathrm{H}$ NMR for major diastereomer $(500 \mathrm{MHz}$, $\left.\mathrm{CDCl}_{3}\right) \delta=7.84(\mathrm{~d}, J=8.3 \mathrm{~Hz}, 2 \mathrm{H}), 7.68(\mathrm{~d}, J=8.3 \mathrm{~Hz}, 2 \mathrm{H}), 7.42(\mathrm{dd}, J=$ 7.1, 1.6 Hz, 1H), 7.28-7.21 (m, 3H), 7.12-7.04 (m, 5H), 6.93-6.88 (m, 2H), 6.53-6.49 (m, 1H), 6.43-6.38 (m, 2H), $5.03(\mathrm{dd}, J=12.5,12.2 \mathrm{~Hz}, 1 \mathrm{H})$, $4.92(\mathrm{dd}, J=15.1,12.1 \mathrm{~Hz}, 1 \mathrm{H}), 4.74(\mathrm{~d}, J=15.9 \mathrm{~Hz}, 1 \mathrm{H}), 4.67$ (dd, $J=$ 15.6, $12.6 \mathrm{~Hz}, 1 \mathrm{H}), 4.28(\mathrm{~d}, J=16.1 \mathrm{~Hz}, 1 \mathrm{H}) \mathrm{ppm} .{ }^{13} \mathrm{C}$ NMR for major diastereomer $\left(126 \mathrm{MHz}, \mathrm{CDCl}_{3}\right) \delta=174.8,143.8,134.4,133.5,130.7$ (q, $\left.{ }^{2} \mathrm{JC}-\mathrm{F}=32.8 \mathrm{~Hz}\right), 129.9,129.3,128.8,128.6,128.6,128.4,128.2,127.3$, $126.5,126.4,126.1$ (q, $\left.{ }^{3} \mathrm{~J}_{\mathrm{C}-\mathrm{F}}=3.8 \mathrm{~Hz}\right), 126.1,123.8\left(\mathrm{q},{ }^{1} \mathrm{~J}_{\mathrm{C}-\mathrm{F}}=271.8 \mathrm{~Hz}\right.$ ), $122.8,110.7,76.5,59.5,50.8,44.0 \mathrm{ppm}$. IR (ATR): $v^{\sim}=2923,1709,1605$ 1547, 1377, 762, $695 \mathrm{~cm}^{-1}$. HPLC: Lux-i-Amylose-1 column, hexane/isopropanol $=95: 5,1.0 \mathrm{~mL} / \mathrm{min}, \quad \lambda=254 \mathrm{~nm}$. Dr 90:10. Major diastereoisomer: $t_{r}=30.29 \mathrm{~min}$ (minor enantiomer), $t_{r}=109.47 \mathrm{~min}$ (major enantiomer) (er 98:2). Minor diastereoisomer: $t_{r}=29.11 \mathrm{~min}$ (major enantiomer), $t_{r}=60.22 \mathrm{~min}$ (minor enantiomer) (er 72:28). HRMS (ESI-QTOF) m/z: $[M+N a]^{+}$Calcd. for $\mathrm{C}_{30} \mathrm{H}_{23} \mathrm{~F}_{3} \mathrm{~N}_{2} \mathrm{NaO}_{3}$ 539.1553; Found 539.1549.

\section{(R)-1-Benzyl-3-(4-methoxyphenyl)-3-((R)-2-nitro-1-}

phenylethyl)indolin-2-one (7la). Product 7la was obtained according to the general procedure, using oxindole $\mathbf{5 l}$, nitrostyrene $\mathbf{6 a}$ and catalyst Thsq-llb. The crude reaction mixture was purified by flash chromatography (Hexane/EtOAc: 8:1 to 4:1) leading to compound 7la as an inseparable mixture of diastereoisomers. White solid (45 $\mathrm{mg}, 0.095 \mathrm{mmol}, 63 \%$ ). $[\alpha]_{D}^{23}=+125.2\left[\left(c=0.20, \mathrm{CHCl}_{3}\right)(\mathrm{dr}=99: 1,>99 \%\right.$ ee for the major diastereomer)]. ${ }^{1} \mathrm{H}$ NMR for major diastereomer $\left(500 \mathrm{MHz}, \mathrm{CDCl}_{3}\right) \delta=$ 7.66-7.61 (m, 2H), 7.48-7.39 (m, 1H), 7.28-7.21 (m, 3H), 7.17-7.10 (m, $1 \mathrm{H}$ ), 7.13-7.05 (m, 4H), 6.94 (ddd, $J=14.6,7.7,1.8 \mathrm{~Hz}, 4 \mathrm{H}$ ), 6.50-6.45 (m, 1H), 6.44-6.39 (m, 2H), 5.05 (dd, $J=12.4,12.3 \mathrm{~Hz}, 1 \mathrm{H}), 4.90(\mathrm{dd}, J=$ $15.1,12.1 \mathrm{~Hz}, 1 \mathrm{H}), 4.80(\mathrm{~d}, J=16.0 \mathrm{~Hz}, 1 \mathrm{H}), 4.80(\mathrm{dd}, J=15.7,12.7 \mathrm{~Hz}$, $1 \mathrm{H}), 4.27(\mathrm{~d}, J=16.1 \mathrm{~Hz}, 1 \mathrm{H}), 3.82(\mathrm{~s}, 3 \mathrm{H}) \mathrm{ppm} .{ }^{13} \mathrm{C}$ NMR for major diastereomer $\left(101 \mathrm{MHz}, \mathrm{CDCl}_{3}\right) \delta=175.8,159.7,143.8,134.7,134.1$, $130.0,129.3,128.8,128.6,128.3,128.3,128.1,127.5,127.1,126.4$, $126.1,122.3,114.6,110.3,76.8,59.0,55.3,50.8,43.9$ ppm. IR (ATR): $\tilde{v}=2965,1709,1605,1543,1510,1489,762,699 \mathrm{~cm}^{-1}$. HPLC: Lux-iAmylose-1 column, hexane/iso-propanol $=85: 15,1.0 \mathrm{~mL} / \mathrm{min}, \lambda=254$ $\mathrm{nm}$. Dr 99:1. Major diastereoisomer: $t_{r}=23.35 \mathrm{~min}$ (minor enantiomer), $t_{r}$ $=103.72 \mathrm{~min}$ (major enantiomer) (er >99:<1). Minor diastereoisomer: $t_{r}=$ $32.53 \mathrm{~min}$ (major enantiomer), $t_{r}=60.55 \mathrm{~min}$ (minor enantiomer) (er 70:30). HRMS (ESI-QTOF) m/z: $[\mathrm{M}+\mathrm{Na}]^{+}$Calcd. for $\mathrm{C}_{30} \mathrm{H}_{26} \mathrm{~N}_{2} \mathrm{NaO}_{4}$ 501.1785; Found 501.1789.

(S)-1-Benzyl-3-methyl-3-((R)-2-nitro-1-phenylethyl)indolin-2-one (7ma). ${ }^{[13 a]}$ Product $7 \mathrm{ma}$ was obtained as a mixture $79: 21$ of diastereoisomers ( $48 \mathrm{mg}, 0.125 \mathrm{mmol}, 83 \%$ ) according to the general procedure, using oxindole $5 \mathrm{~m}$, nitrostyrene $6 \mathrm{a}$ and catalyst Th-sq-Ilb ( $0.03 \mathrm{mmol}, 0.2$ equiv). The major diastereoisomer was isolated by flash chromatography (Hexane/EtOAc: 8:1 to $4: 1$ ) as a colorless oil. [a $]_{D^{23}}=$ $102.0\left[\left(c=0.30, \mathrm{CHCl}_{3}\right)(90 \%\right.$ ee for the major diastereomer $\left.)\right] .{ }^{1} \mathrm{H} \mathrm{NMR}$ for major diastereomer $\left(500 \mathrm{MHz}, \mathrm{CDCl}_{3}\right) \delta=7.23(\mathrm{~m}, 1 \mathrm{H}), 7.20-7.11(\mathrm{~m}$ $7 \mathrm{H}), 7.07(\mathrm{td}, J=7.5,1.1 \mathrm{~Hz}, 1 \mathrm{H}), 6.90(\mathrm{~m}, 2 \mathrm{H}), 6.65(\mathrm{dd}, J=7.9,1.6 \mathrm{~Hz}$, 2H), $6.53(\mathrm{dd}, J=8.1,1.0 \mathrm{~Hz}, 1 \mathrm{H}$ ), $5.14(\mathrm{dd}, J=12.7,4.3 \mathrm{~Hz}, 1 \mathrm{H}$ ), 4.98 (dd, $J=12.7,11.3 \mathrm{~Hz}, 1 \mathrm{H}), 4.88(\mathrm{~d}, J=15.8 \mathrm{~Hz}, 1 \mathrm{H}), 4.44(\mathrm{~d}, J=15.8$ $\mathrm{Hz}, 1 \mathrm{H}$ ), 4.08 (dd, $J=11.4,4.4 \mathrm{~Hz}, 1 \mathrm{H}$ ), 1.57 (s, 3H) ppm. ${ }^{13} \mathrm{C}$ NMR for major diastereomer $\left(101 \mathrm{MHz}, \mathrm{CDCl}_{3}\right) \delta=178.1,142.6,135.0,135.0$,
$130.6,128.9,128.8,128.7,128.3,128.2,127.3,126.7,123.5,122.6$, 109.7, 76.4, 50.7, 50.0, 43.7, $21.7 \mathrm{ppm}$. IR (ATR): $\tilde{v}=2923,1709,1610$, 1552, 1489, 1456, 728, $695 \mathrm{~cm}^{-1}$. HPLC: Lux-Amylose-1 column, hexane/iso-propanol $=90: 10,1.0 \mathrm{~mL} / \mathrm{min}, \lambda=254 \mathrm{~nm}$. Major diastereoisomer: $t_{r}=21.07 \mathrm{~min}$ (minor enantiomer), $t_{r}=29.53 \mathrm{~min}$ (major enantiomer) (er 95:5). HRMS (ESI-QTOF) $\mathrm{m} / \mathrm{z}$ : $[\mathrm{M}+\mathrm{Na}]^{+}$Calcd. for $\mathrm{C}_{24} \mathrm{H}_{22} \mathrm{~N}_{2} \mathrm{NaO}_{3}$ 409.1523; Found 409.1526.

Ethyl 2-((S)-1-Benzyl-3-((R)-2-nitro-1-phenylethyl)-2-oxoindolin-3yl)acetate (7na). Product 7 na was obtained as a mixture 79:21 of diastereoisomers (56 mg, $0.123 \mathrm{mmol}, 82 \%$ ) according to the general procedure, using oxindole $\mathbf{5 n}$, nitrostyrene $\mathbf{6 a}$ and catalyst Th-sq-Ilb (0.03 mmol). The major diastereoisomer was isolated by flash chromatography (Hexane/EtOAc: 8:1 to 4:1) as a white solid; m.p 206$207^{\circ} \mathrm{C}$ from Hexane/EtOAc. $[\alpha]_{D}{ }^{23}=-32.7\left[\left(c=0.40, \mathrm{CHCl}_{3}\right)(88 \%\right.$ ee for the major diastereomer)]. ${ }^{1} \mathrm{H}$ NMR for major diastereomer $(500 \mathrm{MHz}$, $\left.\mathrm{CDCl}_{3}\right) \delta=7.26-7.16(\mathrm{~m}, 7 \mathrm{H}), 7.09-7.04(\mathrm{~m}, 2 \mathrm{H}), 6.92-6.89(\mathrm{~m}, 4 \mathrm{H}), 6.55$ (d, $J=7.8 \mathrm{~Hz}, 1 \mathrm{H}$ ), 4.96 (dd, $J=12.8,4.4 \mathrm{~Hz}, 1 \mathrm{H}), 4.88$ (dd, $J=12.8$, $11.0 \mathrm{~Hz}, 1 \mathrm{H}), 4.80(\mathrm{~d}, J=15.8 \mathrm{~Hz}, 1 \mathrm{H}), 4.54(\mathrm{~d}, J=15.8 \mathrm{~Hz}, 1 \mathrm{H}), 3.99$ (dd, $J=11.0,4.4 \mathrm{~Hz}, 1 \mathrm{H}), 3.90(\mathrm{~m}, 1 \mathrm{H}), 3.80(\mathrm{~m}, 1 \mathrm{H}), 3.16(\mathrm{~d}, J=16.0$ $\mathrm{Hz}, 1 \mathrm{H}), 3.02(\mathrm{~d}, J=16.0 \mathrm{~Hz}, 1 \mathrm{H}), 0.94(\mathrm{t}, J=7.1 \mathrm{~Hz}, 3 \mathrm{H}) \mathrm{ppm} .{ }^{13} \mathrm{C}$ NMR for major diastereomer $\left(101 \mathrm{MHz}, \mathrm{CDCl}_{3}\right) \delta=177.0,168.7,144.1,135.3$, 134.0, 129.2, 128.6, 128.5, 128.3, 128.1, 127.4, 127.0, 123.3, 122.4, 109.6, 76.1, 60.8, 51.8, 50.0, 44.2, 39.2, $13.8 \mathrm{ppm}$. IR (ATR): $\tilde{v}=2923$, 1701, 1610, 1556, 1489, 1468, 1456, $699 \mathrm{~cm}^{-1}$. HPLC: Lux-i-Amylose-1 column, hexane/iso-propanol $=90: 10,1.0 \mathrm{~mL} / \mathrm{min}, \lambda=254 \mathrm{~nm}$. Major diastereoisomer: $\mathrm{t}_{\mathrm{r}}=42.97 \mathrm{~min}$ (minor enantiomer), $\mathrm{t}_{\mathrm{r}}=56.29 \mathrm{~min}$ (major enantiomer) (er 94:6). HRMS (ESI-QTOF) $\mathrm{m} / \mathrm{z}:[\mathrm{M}+\mathrm{Na}]^{+}$Calcd. for $\mathrm{C}_{27} \mathrm{H}_{26} \mathrm{~N}_{2} \mathrm{NaO}_{5}$ 481.1734; Found 481.1736.

(R)-1-Benzyl-3-((R)-1-(4-chlorophenyl)-2-nitroethyl)-3-phenylindolin2-one (7bb). Product $\mathbf{7 b b}$ was obtained according to the general procedure, using oxindole $\mathbf{5 b}$, nitrostyrene $\mathbf{6 b}$ and catalyst Th-sq- $\mathbf{l l} \mathbf{b}$. The crude reaction mixture was purified by flash chromatography (Hexane/EtOAc: 8:1 to 4:1) leading to compound $\mathbf{7 b b}$ as an inseparable mixture of diastereoisomers. White solid (59 mg, $0.12 \mathrm{mmol}, 81 \%)$. [a $]_{\mathrm{D}}^{23}$ $=+86.1\left[\left(c=0.70, \mathrm{CHCl}_{3}\right) \quad(\mathrm{dr}=94: 6,98 \%\right.$ ee for the major diastereomer)]. ${ }^{1} \mathrm{H}$ NMR for major diastereomer $\left(500 \mathrm{MHz}, \mathrm{CDCl}_{3}\right) \delta=$ 7.71-7.66 (m, 2H), 7.46-7.41 (m, 3H), 7.41-7.36 (m, 1H), 7.32-7.25 (m, $2 \mathrm{H}), 7.20-7.15(\mathrm{~m}, 3 \mathrm{H}), 7.06(\mathrm{~d}, J=8.7 \mathrm{~Hz}, 2 \mathrm{H}), 6.86(\mathrm{~d}, J=8.6 \mathrm{~Hz}, 2 \mathrm{H})$, 6.58-6.55 (m, 1H), 6.48 (dd, $J=7.5,2.0 \mathrm{~Hz}, 2 \mathrm{H}), 4.99$ (dd, $J=12.3,12.2$ $\mathrm{Hz}, 1 \mathrm{H}), 4.92(\mathrm{dd}, J=14.8,12.2 \mathrm{~Hz}, 1 \mathrm{H}), 4.89(\mathrm{~d}, J=16.0 \mathrm{~Hz}, 1 \mathrm{H}), 4.75$ (dd, $J=14.8,12.3 \mathrm{~Hz}, 1 \mathrm{H}), 4.26\left(\mathrm{~d}, J=16.0 \mathrm{~Hz}, 1 \mathrm{H}\right.$ ) ppm. ${ }^{13} \mathrm{C}$ NMR for major diastereomer $\left(101 \mathrm{MHz}, \mathrm{CDCl}_{3}\right) \delta=175.3,143.8,136.0,134.5$, $134.4,132.6,130.7,129.6,129.3,128.6,127.5,127.4,126.9,126.5$, 126.2, 122.5, 110.5, 76.6, 59.3, 50.1, $44.0 \mathrm{ppm}$. IR (ATR): $\tilde{v}=2923$, 1714, 1610, 1552, 1489, 1464, 728, $695 \mathrm{~cm}^{-1}$. HPLC: Lux-i-Amylose-1 column, hexane/iso-propanol $=95: 5,1.0 \mathrm{~mL} / \mathrm{min}, \lambda=254 \mathrm{~nm}$. Dr 94:6. Major diastereoisomer: $t_{r}=42.50 \mathrm{~min}$ (minor enantiomer), $t_{r}=48.72 \mathrm{~min}$ (major enantiomer) (er 99:1). Minor diastereoisomer: $t_{r}=28.10 \mathrm{~min}$ (major enantiomer), $t_{r}=59.88 \mathrm{~min}$ (minor enantiomer) (er 77:23). HRMS (ESI-QTOF) m/z: $[M+N a]^{+}$Calcd. for $\mathrm{C}_{29} \mathrm{H}_{23} \mathrm{ClN}_{2} \mathrm{NaO}_{3}$ 505.1293; Found 505.1289 .

(R)-1-Benzyl-3-((R)-2-nitro-1-(4-(trifluoromethyl)phenyl)ethyl)-3phenylindolin-2-one (7bc). Product $7 \mathrm{bc}$ was obtained according to the general procedure, using oxindole $\mathbf{5 b}$, nitrostyrene $\mathbf{6 c}$ and catalyst Th- 
sq-Illb. The crude reaction mixture was purified by flash chromatography (Hexane/EtOAc: 8:1 to 4:1) leading to compound $\mathbf{7 b c}$ as an inseparable mixture of diastereoisomers. White solid $(75 \mathrm{mg}, 0.145 \mathrm{mmol}, 97 \%)$. $[\alpha]_{D}^{23}=+51.0\left[\left(c=0.10, \mathrm{CHCl}_{3}\right)(\mathrm{dr}=93: 7,98 \%\right.$ ee for the major diastereomer)]. ${ }^{1} \mathrm{H}$ NMR for major diastereomer $\left(500 \mathrm{MHz}, \mathrm{CDCl}_{3}\right) \delta=$ 7.71-7.67 (m, 2H), 7.45 (ddd, $J=7.7,6.6,1.5 \mathrm{~Hz}, 3 \mathrm{H}), 7.41-7.38(\mathrm{~m}, 1 \mathrm{H})$ $7.35(\mathrm{~d}, J=8.2 \mathrm{~Hz}, 2 \mathrm{H}), 7.29(\mathrm{dd}, J=4.3,1.5 \mathrm{~Hz}, 1 \mathrm{H}), 7.28-7.25(\mathrm{~m}, 1 \mathrm{H})$, 7.19-7.14 (m, 1H), 7.12-7.05 (m, 4H), 6.60-6.56 (m, 1H), 6.56-6.52 (m, 2H), 5.04 (dd, $J=12.2,10.9 \mathrm{~Hz}, 1 \mathrm{H}), 5.04$ (dd, $J=16.5,10.9 \mathrm{~Hz}, 1 \mathrm{H}$ ), $4.78(\mathrm{dd}, J=16.5,12.1 \mathrm{~Hz}, 1 \mathrm{H}), 4.78(\mathrm{~d}, J=15.9 \mathrm{~Hz}, 1 \mathrm{H}), 4.30(\mathrm{~d}, J=$ $15.9 \mathrm{~Hz}, 1 \mathrm{H}) \mathrm{ppm} .{ }^{13} \mathrm{C} \mathrm{NMR}$ for major diastereomer $\left(101 \mathrm{MHz}, \mathrm{CDCl}_{3}\right) \delta$ $=175.2,143.7,138.3,135.9,134.5,130.5\left(\mathrm{q},{ }^{2} \mathrm{~J}_{\mathrm{C}-\mathrm{F}}=32.6 \mathrm{~Hz}\right), 129.8$, $129.4,128.7,128.6,127.5,127.5,126.7,126.5,126.2,125.2\left(q,{ }^{3} J_{C-F}=\right.$ $3.7 \mathrm{~Hz}$ ), 125.2, 124.5 (q, $\left.{ }^{1} \mathrm{~J}_{\mathrm{C}-\mathrm{F}}=271.9 \mathrm{~Hz}\right), 122.6,110.5,76.4,59.2,50.3$, $44.1 \mathrm{ppm}$. IR (ATR): $\tilde{v}=2927,1709,1610,1556,1489,1468,749,695$ $\mathrm{cm}^{-1}$. HPLC: Lux-i-Amylose-1 column, hexane/iso-propanol $=97: 3,1.0$ $\mathrm{mL} / \mathrm{min}, \lambda=254 \mathrm{~nm}$. Dr 93:7. Major diastereoisomer: $t_{r}=69.64 \mathrm{~min}$ (minor enantiomer), $t_{r}=75.63 \mathrm{~min}$ (major enantiomer) (er 99:1). Minor diastereoisomer: $t_{r}=31.56 \mathrm{~min}$ (major enantiomer), $t_{r}=88.51 \mathrm{~min}$ (minor enantiomer) (er 86:14). HRMS (ESI-QTOF) $\mathrm{m} / \mathrm{z}$ : $[\mathrm{M}+\mathrm{Na}]^{+}$Calcd. for $\mathrm{C}_{30} \mathrm{H}_{23} \mathrm{~F}_{3} \mathrm{~N}_{2} \mathrm{NaO}_{3}$ 539.1553; Found 539.1555.

(R)-1-Benzyl-3-((R)-1-(4-methoxyphenyl)-2-nitroethyl)-3-

phenylindolin-2-one (7bd). Product $7 \mathrm{bd}$ was obtained according to the general procedure, using oxindole $5 \mathbf{b}$, nitrostyrene $\mathbf{6 d}$ and catalyst Thsq-llb. The crude reaction mixture was purified by flash chromatography (Hexane/EtOAc: 8:1 to 4:1) leading to compound $7 \mathbf{b d}$ as an inseparable mixture of diastereoisomers. White solid (58 mg, $0.12 \mathrm{mmol}, 81 \%)$. [a $]_{\mathrm{D}}{ }^{23}$ $=+74.0\left[\left(c=0.60, \mathrm{CHCl}_{3}\right)(\mathrm{dr}=92: 8,94 \%\right.$ ee for the major diastereomer)]. ${ }^{1} \mathrm{H}$ NMR for major diastereomer $\left(500 \mathrm{MHz}, \mathrm{CDCl}_{3}\right) \delta=$ $7.70(\mathrm{dd}, J=8.3,1.4 \mathrm{~Hz}, 2 \mathrm{H}), 7.43(\mathrm{td}, J=7.5,6.8,1.5 \mathrm{~Hz}, 3 \mathrm{H}), 7.39-$ $7.37(\mathrm{~m}, 1 \mathrm{H}), 7.28-7.23(\mathrm{~m}, 2 \mathrm{H}), 7.17-7.12(\mathrm{~m}, 1 \mathrm{H}), 7.10-7.05(\mathrm{~m}, 2 \mathrm{H})$, 6.86-6.81 (m, 2H), 6.65-6.60 (m, 2H), $6.51(\mathrm{dd}, J=7.8,1.3 \mathrm{~Hz}, 1 \mathrm{H}), 6.44-$ $6.40(\mathrm{~m}, 2 \mathrm{H}), 5.00(\mathrm{dd}, J=12.3,12.3 \mathrm{~Hz}, 1 \mathrm{H}), 4.90(\mathrm{dd}, J=15.2,12.3 \mathrm{~Hz}$ $1 \mathrm{H}), 4.90(\mathrm{~d}, J=16.1 \mathrm{~Hz}, 1 \mathrm{H}), 4.75(\mathrm{dd}, J=15.2,12.3 \mathrm{~Hz}, 1 \mathrm{H}), 4.25$ (d, $J$ $=16.1 \mathrm{~Hz}, 1 \mathrm{H}), 3.74(\mathrm{~s}, 3 \mathrm{H}) \mathrm{ppm} \cdot{ }^{13} \mathrm{C}$ NMR for major diastereomer $(101$ $\left.\mathrm{MHz}, \mathrm{CDCl}_{3}\right) \delta=175.6,159.5,143.9,136.4,134.6,130.5,129.4,129.2$, $128.4,128.4,127.6,127.4,127.2,126.5,126.2,125.8,122.4,113.8$, $110.4,77.0,59.7,55.0,50.1,43.9 \mathrm{ppm}$. IR (ATR): $v^{2}=2915,1709,1610$, 1552, 1514, 1468, 741, 691, $649 \mathrm{~cm}^{-1}$. HPLC: Lux-i-Amylose-1 column, hexane/iso-propanol $=95: 5,1.0 \mathrm{~mL} / \mathrm{min}, \lambda=254 \mathrm{~nm}$. Dr 92:8. Major diastereoisomer: $t_{r}=51.15 \mathrm{~min}$ (minor enantiomer), $t_{r}=54.42 \mathrm{~min}$ (major enantiomer) (er 97:3). Minor diastereoisomer: $t_{r}=35.17 \mathrm{~min}$ (major enantiomer), $t_{r}=64.61 \mathrm{~min}$ (minor enantiomer) (er 73:27). HRMS (ESIQTOF) $\mathrm{m} / \mathrm{z}:[\mathrm{M}+\mathrm{Na}]^{+}$Calcd. for $\mathrm{C}_{30} \mathrm{H}_{26} \mathrm{~N}_{2} \mathrm{NaO}_{4}$ 501.1785; Found 501.1787.

(R)-1-Benzyl-3-((R)-1-(naphthalen-2-yl)-2-nitroethyl)-3-phenylindolin2-one (7be). Product 7 be was obtained according to the general procedure, using oxindole $5 \mathbf{b}$, nitrostyrene $\mathbf{6 e}$ and catalyst $\mathbf{T h}$-sq-Ilb. The crude reaction mixture was purified by flash chromatography (Hexane/EtOAc: 8:1 to 4:1) leading to compound 7be as an inseparable mixture of diastereoisomers. Yellow oil (73 mg, $0.147 \mathrm{mmol}, 98 \%)$. [a] $]^{23}$ $=+39.7\left[\left(c=0.90, \mathrm{CHCl}_{3}\right)(\mathrm{dr}=93: 7,96 \%\right.$ ee for the major diastereomer)]. ${ }^{1} \mathrm{H}$ NMR for major diastereomer $\left(500 \mathrm{MHz}, \mathrm{CDCl}_{3}\right) \delta=$ 7.81-7.76 (m, 3H), 7.62 (d, $J=8.2 \mathrm{~Hz}, 1 \mathrm{H}), 7.56-7.40(\mathrm{~m}, 8 \mathrm{H}), 7.32-7.25$ (m, 2H), 6.97 (dd, $J=8.8,1.8 \mathrm{~Hz}, 1 \mathrm{H}), 6.87(\mathrm{t}, J=7.5 \mathrm{~Hz}, 1 \mathrm{H}), 6.49-6.41$ (m, 3H), 6.12 (dd, $J=7.9,1.3 \mathrm{~Hz}, 2 \mathrm{H}$ ), 5.17 (dd, $J=12.2,11.5 \mathrm{~Hz}, 1 \mathrm{H}$ ), 5.17 (dd, $J=12.2,10.8 \mathrm{~Hz}, 1 \mathrm{H}$ ), $4.85(\mathrm{~d}, J=16.6 \mathrm{~Hz}, 1 \mathrm{H}$ ), 4.85 (dd, $J=$ $11.5,10.8 \mathrm{~Hz}, 1 \mathrm{H}), 4.13(\mathrm{~d}, J=16.1 \mathrm{~Hz}, 1 \mathrm{H}) \mathrm{ppm} .{ }^{13} \mathrm{C}$ NMR $(101 \mathrm{MHz}$, $\left.\mathrm{CDCl}_{3}\right) \delta=175.5,143.9,136.4,134.2,133.2,133.1,131.6,129.5,129.4$, $129.3,128.5,128.3,128.1,128.0,127.6,127.5,127.3,127.0,126.4$, $126.3,126.3,126.2,126.0,122.5,110.5,77.0,59.6,50.9,43.9$ ppm. IR (ATR): $v=3060,2919,1709,1609,1552,1489,728,695 \mathrm{~cm}^{-1}$. HPLC: Lux-i-Amylose-1 column, hexane/iso-propanol $=95: 5,1.0 \mathrm{~mL} / \mathrm{min}, \lambda=$ $254 \mathrm{~nm}$. Dr 93:7. Major diastereoisomer: $t_{r}=54.60 \mathrm{~min}$ (minor enantiomer), $t_{r}=90.02 \mathrm{~min}$ (major enantiomer) (er 98:2). Minor diastereoisomer: $t_{r}=50.23 \mathrm{~min}$ (major enantiomer), $t_{r}=111.32 \mathrm{~min}$ (minor enantiomer) (er 78:22). HRMS (ESI-QTOF) m/z: $[M+N a]^{+}$Calcd. for $\mathrm{C}_{33} \mathrm{H}_{26} \mathrm{~N}_{2} \mathrm{NaO}_{3}$ 521.1836; Found 521.1836.

(R)-1-Benzyl-3-((R)-1-(furan-2-yl)-2-nitroethyl)-3-phenylindolin-2-one (7bf). Product $7 \mathbf{b f}$ was obtained according to the general procedure, using oxindole $5 \mathbf{b}$, nitrostyrene $6 f$ and catalyst Th-sq-llb. The crude reaction mixture was purified by flash chromatography (Hexane/EtOAc: $8: 1$ to $4: 1)$ leading to compound $7 \mathbf{b f}$ as an inseparable mixture of diastereoisomers. White solid (48 mg, $0.11 \mathrm{mmol}, 73 \%$ ). [a] ${ }^{23}=+144.3$ $\left[\left(c=0.60, \mathrm{CHCl}_{3}\right)(\mathrm{dr}=84: 16,94 \%\right.$ ee for the major diastereomer $\left.)\right] .{ }^{1} \mathrm{H}$ NMR $\left(500 \mathrm{MHz}, \mathrm{CDCl}_{3}\right) \delta=7.61(\mathrm{~d}, J=7.6 \mathrm{~Hz}, 2 \mathrm{H}), 7.45-7.21(\mathrm{~m}, 9 \mathrm{H})$, $7.03(\mathrm{~d}, J=2.0 \mathrm{~Hz}, 1 \mathrm{H}$ ), 6.93 (dd, $J=6.6,3.0 \mathrm{~Hz}, 2 \mathrm{H}), 6.64$ (d, $J=7.8$ $\mathrm{Hz}, 1 \mathrm{H}), 6.10(\mathrm{dd}, J=3.3,1.8 \mathrm{~Hz}, 1 \mathrm{H}), 5.96(\mathrm{~d}, J=3.3 \mathrm{~Hz}, 1 \mathrm{H}), 5.09(\mathrm{dd}$, $J=12.6,10.8 \mathrm{~Hz}, 1 \mathrm{H}$ ), 5.09 (dd, $J=12.6,11.4 \mathrm{~Hz}, 1 \mathrm{H}), 4.84(\mathrm{~d}, J=15.8$ $\mathrm{Hz}, 1 \mathrm{H}), 4.66$ (dd, $J=11.4,10.7 \mathrm{~Hz}, 1 \mathrm{H}), 4.58(\mathrm{~d}, J=15.8 \mathrm{~Hz}, 1 \mathrm{H}) \mathrm{ppm}$. ${ }^{13} \mathrm{C} \mathrm{NMR}\left(101 \mathrm{MHz}, \mathrm{CDCl}_{3}\right) \delta=175.8,148.5,143.3,142.5,135.9,135.0$, 129.3, 129.2, 128.6, 128.5, 127.5, 127.4, 127.4, 127.1, 126.1, 122.4 110.2, 110.0, 109.5, 75.0, 58.8, 44.6, $44.1 \mathrm{ppm}$. IR (ATR): $\tilde{v}=2919$, 1714, 1610, 1547, 1489, 745, $695 \mathrm{~cm}^{-1}$. HPLC: Lux-i-Amylose-1 column, hexane/iso-propanol $=97: 3,1.0 \mathrm{~mL} / \mathrm{min}, \lambda=254 \mathrm{~nm}$. Dr 84:16. Major diastereoisomer: $t_{r}=46.00 \mathrm{~min}$ (minor enantiomer), $t_{r}=199.72 \mathrm{~min}$ (major enantiomer) (er 97:3). Minor diastereoisomer: $t_{r}=75.12 \mathrm{~min}$ (major enantiomer), $t_{r}=79.92 \mathrm{~min}$ (minor enantiomer) (er 64:36). HRMS (ESI-QTOF) m/z: $[M+N a]^{+}$Calcd. for $\mathrm{C}_{27} \mathrm{H}_{22} \mathrm{~N}_{2} \mathrm{NaO}_{4}$ 461.1472; Found 461.1475 .

\section{(3S,5'R)-1-Benzyl-5'-phenylspiro[indoline-3,4'-piperidine]-2,2'-dione}

(8). A mixture of $\mathrm{Zn}$ powder ( $340 \mathrm{mg}, 5.2 \mathrm{mmol}, 20$ equivalents) and $7 \mathrm{na}$ (119 mg, $0.26 \mathrm{mmol})$ in HOAc $(1.8 \mathrm{~mL})$ was heated at $70^{\circ} \mathrm{C}$ overnight. When the reaction was finished, the mixture was filtered through a pad of celite and the filtrate was concentrated under vacuum. The mixture was redissolved in $\mathrm{DCM}$ and then an aqueous solution of $\mathrm{NaHCO}_{3}$ was added until basic $\mathrm{pH}$. The product was extracted with DCM $(3 \times 10 \mathrm{~mL})$. The organic layer was washed with brine, dried over anhydrous $\mathrm{MgSO}_{4}$, and concentrated under reduced pressure. The compound was purified by flash column chromatography on silica gel (EtOAc/MeOH 40:1 to 15:1) affording compound 8 as a white solid ( $83 \mathrm{mg}, 0.216 \mathrm{mmol}, 83 \%$ ); m.p $166-168{ }^{\circ} \mathrm{C} .[\alpha]_{D}{ }^{23}=-131.7\left[\left(c=1.00, \mathrm{CHCl}_{3}\right)(88 \%\right.$ ee for the major diastereomer)]. ${ }^{1} \mathrm{H}$ NMR for major diastereomer $\left(500 \mathrm{MHz}, \mathrm{CDCl}_{3}\right) \delta=$ 7.53 (dd, $J=7.4,1.4 \mathrm{~Hz}, 1 \mathrm{H}), 7.28$ (br s, 1H), $7.24(\mathrm{~m}, 1 \mathrm{H}), 7.19$ (td, $J=$ 7.7, $1.3 \mathrm{~Hz}, 1 \mathrm{H}), 7.16-7.03(\mathrm{~m}, 6 \mathrm{H}), 6.77(\mathrm{~d}, J=7.2 \mathrm{~Hz}, 2 \mathrm{H}), 6.46(\mathrm{~d}, J=$ $7.6 \mathrm{~Hz}, 1 \mathrm{H}), 6.35(\mathrm{~d}, J=7.4 \mathrm{~Hz}, 2 \mathrm{H}), 5.03(\mathrm{~d}, J=16.0 \mathrm{~Hz}, 1 \mathrm{H}), 4.25(\mathrm{~d}, J$ $=16.0 \mathrm{~Hz}, 1 \mathrm{H}), 4.01(\mathrm{t}, J=11.9 \mathrm{~Hz}, 1 \mathrm{H}), 3.77-3.62(\mathrm{~m}, 2 \mathrm{H}), 3.21(\mathrm{~d}, J=$ $17.5 \mathrm{~Hz}, 1 \mathrm{H}), 2.44(\mathrm{~d}, J=17.5 \mathrm{~Hz}, 1 \mathrm{H}) .{ }^{13} \mathrm{C}$ NMR for major diastereomer 
$\left(126 \mathrm{MHz}, \mathrm{CDCl}_{3}\right) \delta=176.6,170.3,142.8,135.7,134.7,129.0,128.6$, 128.5, 128.5, 128.1, 127.1, 126.3, 124.3, 123.0, 109.8, 51.3, 45.2, 44.5 43.8, 39.3 ppm. IR (ATR): $\tilde{v}=3240,3060,3037,2923,1709,1664,1614$ $758,728,695 \mathrm{~cm}^{-1}$. HPLC: Lux-Amylose-1 column, hexane/iso-propanol $=90: 10,1.0 \mathrm{~mL} / \mathrm{min}, \lambda=254 \mathrm{~nm} . \mathrm{tr}_{r}=47.04 \mathrm{~min}$ (major enantiomer), $\mathrm{tr}_{\mathrm{r}}=$ $106.17 \mathrm{~min}$ (minor enantiomer) (er 94:6). HRMS (ESI-QTOF) m/z: $[M+\mathrm{Na}]^{+}$Calcd. for $\mathrm{C}_{25} \mathrm{H}_{22} \mathrm{~N}_{2} \mathrm{NaO}_{2}$ 405.1573; Found 405.1577.

\section{Acknowledgments}

Authors thank Junta de Castilla y León (Projects FEDERVA115P17, and VA149G18) for financial support. The aid in the X-Ray diffraction determination provided by Dr. R. García Rodríguez is also acknowledged.

Keywords: Asymmetric catalysis $\cdot$ Michael addition • Oxindoles - Thiosquaramides $\cdot$ Spiro compounds

[1] For selected reviews see: a) Z. Zhang, P. R. Schreiner, Chem. Soc Rev. 2009, 38, 1187. b) S. J. Connon, Chem. Eur. J. 2006, 12, 5418.

[2] For selected reviews see: a) G. Koutoulogenis, N. Kaplaneris, C. G. Kokotos, Beilstein J. Org. Chem. 2016, 12, 462. b) X. Fang, C.-J. Wang Chem. Commun. 2015, 51, 1185. c) F. Giacalone, M. Gruttadauria, P. Agrigento, R. Noto, Chem. Soc. Rev. 2012, 41, 2406. d) C. Palomo, M Oiarbide, R. López, Chem. Soc. Rev. 2009, 38, 632.

[3] For selected reviews see: a) B.-L. Zhao, J.-H. Li, D.-M. Du, Chem. Rec. 2017, 17, 1. b) M. Tsakos, C. G. Kokotos. Tetrahedron 2013, 69, 10199 c) J. Alemán, A. Parra, H. Jiang, K. A. Jørgensen, Chem. Eur. J. 2011 17, 6890. d) R. I. Storer, C. Aciro, L. H. Jones, Chem. Soc. Rev. 2011 40, 2330.

[4] G. Maahs, P. Hegenberg. Angew. Chem. Int. Ed. 1966, 5, 888.

[5] T. Lu, S. E. Wheeler, Chem. Eur. J. 2013, 19, 15141

[6] For recent references see: a) R. B. P. Elmes, N. Busschaert, D. D. Czech, P. A. Gale, K. A. Jolliffe, Chem. Commun. 2015, 51, 10107. b) N. Busschaert, R. B. P. Elmes, D. D. Czech, X. Wu, I. L. Kirby, E. M. Peck, K. D. Hendzel, S. K. Shaw, B. Chan, B. D. Smith, K. A. Jolliffe, P. A. Gale, Chem. Sci. 2014, 5, 3617.

[7] G. R. Frauenhoff, F. Takasagawa, D. H. Busch, Inorg. Chem. 1992, 31, 4002.

[8] For the first references see: a) M. Rombola, V. H. Rawal, Org. Lett. 2018, 20, 514. b) M. Rombola, C. S. Sumaria, T. D. Montgomery, V. H. Rawal, J. Am. Chem. Soc. 2017, 139, 5297. Novel thiosquaramides have been reported during the writing this paper, see: c) S. Nagy, G Dargó, P. Kisszékelyi, Z. Fehér, A. Simon, J. Barabás, T. Höltzl, B. Mátravölgyi, L. Kárpáti, L. Drahos, P. Huszthy, J. Kupai, New. J. Chem. 2019, 43, 5948. d) M. Yang, M.; C. Chen, X. Yi, Y. Li, X. Wu, Q. Li, S Ban, Org. Biomol. Chem. 2019, 17, 2883.

[9] a) J. M. Andrés, A. Maestro, M. Valle, I. Valencia, R. Pedrosa, ACS Omega. 2018, 3, 16591. b) J. M. Andrés, A. Maestro, M. Valle, R. Pedrosa, J. Org. Chem. 2018, 83, 5546. c) J. M. Andrés, M. González, A. Maestro, D. Naharro, R. Pedrosa, Eur. J. Org. Chem. 2017, 2683

[10] C. V. Galliford, K. A. Scheidt, Angew. Chem. Int. Ed. 2007, 46, 8748.

[11] B. Trost, M. K. Brennan, Synthesis 2009, 3003.

[12] a) X. Dou, B. Zhou, W. Yao, F. Zhong, C. Jiang, Y. Lu, Org. Lett. 2013 15, 4920. b) K. Albertshofer, B. Tan, C. F. Barbas III, Org. Lett. 2012 14, 1834. c) M. Ding, F. Zhou, Z.-Q. Qian, J. Zhou, Org. Biomol. Chem. 2010, 8, 2912.

[13] a) C. Reiter, S. López-Molina, B. Schmid, C. Neiss, A. Görling, S. B. Tsogoeva, ChemCatChem 2014, 6, 1324. b) Y.-M. Li, X. Li, F.-Z. Peng Z.-Q. Li, S.-T. Wu, Z.-W. Sun, H.-B. Zhang, Z.-H. Shao, Org. Lett. 2011 13, 6200. c) X. Li, Y.-M. Li, F.-Z. Peng, S.-T. Wu, Z.-Q. Li, Z.-W. Sun, H.-B. Zhang, Z.-H. Shao, Org. Lett. 2011, 13, 6160. d) X. Li, B. Zhang, Z.-G. Xi, S. Luo, J.-P. Cheng, Adv. Synth. Catal. 2010, 352, 416. e) T. Bui, S. Syed, C. F. Barbas III, J. Am. Chem. Soc. 2009, 131, 8758.
[14] B.-D. Cui, W.-Y. Han, Z.-J. Wu, X.-M. Zhang, W.-C. Yuan, J. Org. Chem. 2013, 78, 8833

[15] L. Zou, X. Bao, Y. Ma, Y. Song, J. Qu, B. Wang, Chem Commun. 2014, 50,5760 .

[16] M. Ding, F. Zhou, Y.-L. Liu, C.-H. Wang, X.-L. Zhao, J. Zhou, Chem. Sci. 2011, 2, 2035.

[17] X.-L. Liu, Z.-J. Wu, X.-L. Du, X.-M. Zhang, W.-C. Yuan, J. Org. Chem. 2011, 76, 4008 .

[18] R. He, S. Shirakawa, K. Maruoka, J. Am. Chem. Soc. 2009, 131, 16620.

[19] C. Wang, X. Yang, D. Enders, Chem. Eur. J. 2012, 18, 4832

[20] W. Yang, J. Wang, D.-M. Du, Tetrahedron: Asymmetry 2012, 23, 972.

[21] a) M.-X. Zhao, F.-H. Ji, X.-L. Zhao, Z.-Z. Han, M. Shi, Eur. J. Org. Chem. 2014, 644. b) A. Noole, I. Järving, F. Werner, M. Lopp, A. Malkov, T. Kanger, Org. Lett. 2012, 14, 4922.

[22] Y.-H. Liao, X.-L. Liu, Z.-J. Wu, L.-F. Cun, X.-M. Zhang, W.-C. Yuan, Org. Lett. 2010, 12, 2896.

[23] X. Li, Z.-G. Xi, S. Luo, J.-P. Cheng, Org. Biomol. Chem. 2010, 8, 77.

[24] J. M. Andrés, A. Maestro, P. Rodríguez-Ferrer, I. Simón, R. Pedrosa, ChemistrySelect 2016, 1, 5057.

[25] J. M. Andrés, J. Losada, A. Maestro, P. Rodríguez-Ferrer, R. Pedrosa, J. Org. Chem. 2017, 82, 8444.

[26] J. M. Andrés, R. Manzano, R. Pedrosa, Chem. Eur. J. 2008, 14, 5116.

[27] CCDC 1913525 contains the crystallographic data for compound 7ha These data can be obtained free of charge from the Cambridge Crystallographic Data Center via www.ccdc.cam.ac.uk/data request/cif.

[28] For a recent example see: S. Jayakumar, K. Louven, C. Strohmann, K Kumar, Angew. Chem. Int. Ed. 2017, 56, 15945.

[29] For a recent review see: B. Yu, D.-Q. Yu, H.-M. Liu, Eur. J. Med. Chem. 2015, 97, 673

[30] X. Chen, W. Zhu, W. Qian, E. Feng, Y. Zhou, J. Wang, H. Jiang, Z.-J. Yao, H. Liu, H. Adv. Synth. Catal. 2012, 354, 2151.

[31] M. B. Trost, J. Xie, J. D. Sieber, J. Am. Chem. Soc. 2011, 133, 20611.

[32] T. Ishimaru, N. Shibata, J. Nagai, S. Nakamura, T. Toru, S. Kanemasa, J. Am. Chem. Soc. 2006, 128, 16488.

[33] W.-T. Chen, L.-H. Gao, W.-H. Bao, W.-T. Wei, J. Org. Chem. 2018, 83 11074.

[34] J. E. Thomson, A. F. Kyle, K. A. Gallagher, P. Lenden, C. Concellón, K. A. Morrill, A. J. Miller, C. Joannesse, A. M. Z. Slawin, A. D. Smith, Synthesis 2008, 17, 2805.

[35] S.-H. Cao, X.-C. Zhang, Y. Wei, M. Shi, Eur. J. Org. Chem. 2011, 2668.

[36] A. Kamal, R. Mahesh, V. L. Nayak, K. S. Babu, G. B. Kumar, A. B. Shaik, J. S. Kapure, A. Alarifi, Eur. J. Med. Chem. 2016, 108, 476.

[37] Z. Wu, X. Fang, Y. Leng, H. Yao, A. Lin, Adv. Synth. Catal. 2018, 360 1289.

[38] F. Auria-Luna, E. Marqués-López, S. Mohammadi, R. Heiran, R. P. Herrera, Molecules 2015, 20, 15807.

[39] V. Laina-Martín, J. Humbrías-Martín, J. A. Fernández-Salas, J. Alemán, J. Chem. Commun. 2018, 54, 2781.

[40] M. F. Abo-Ashoura, W. M. Eldehnab, R.F. George, M.M. Abdel-Azizd, M.M. Elaasser, N. M. Abdel Gawad, A. Gupta, S. Bhakta, S. M. AbouSeric, Eur. J. Med. Chem 2018, 160, 49. 
Entry for the Table of Contents (Please choose one layout)

Layout 1:

\section{FULL PAPER}

Text for Table of Contents (about 350 characters)

*one or two words that highlight the emphasis of the paper or the field of the study

\section{Key Topic*}

Author(s), Corresponding Author(s)*

Page No. - Page No.

Title

( width: $5.5 \mathrm{~cm}$; max. height: $5.0 \mathrm{~cm}$; NOTE: the final letter height should not be less than $2 \mathrm{~mm}$.))

Layout 2:

\section{FULL PAPER}

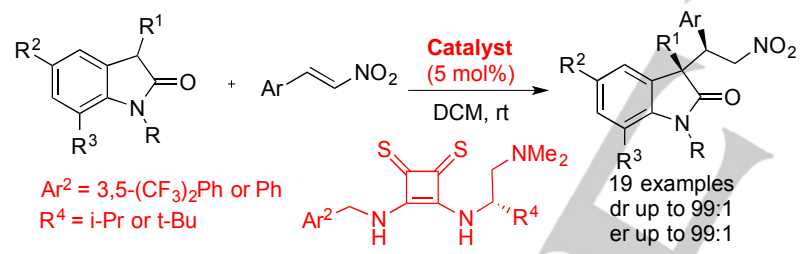

Four novel chiral bifunctional thiosquaramides have been prepared from cyclopentyl dithiosquarates and diamines derived from natural L-Valine and L-tert-Leucine. The novel thiosquaramides have been tested as organocatalyst in the nitro-Michael addition of 3-substituted oxindoles to different $\beta$-aryl substituted nitroalkenes. The reaction occurred easily in high yields and excellent stereoselectivities.
Asymmetric Organocatalysis

Patricia Rodríguez-Ferrer, ${ }^{[a]}$ Daniel Naharro, ${ }^{[a]}$ Alicia Maestro, ${ }^{[a]}$ José M. Andrés, ${ }^{*[a]}$ and Rafael Pedrosa. ${ }^{*[a]}$

Page No. - Page No.

Chiral Bifunctional Thiosquaramides as Organocatalysts in the Synthesis of Enantioenriched 3,3-Disubstituted Oxindoles 